\title{
Noncommutative fermions and quarkonia decays
}

\author{
Carlos Tamarit ${ }^{1}$ and Josip Trampetić ${ }^{2}$ \\ ${ }^{1}$ Departamento de Física Teórica I, Facultad de Ciencias Físicas, \\ Universidad Complutense de Madrid, 28040 Madrid, Spain \\ ${ }^{2}$ Theoretical Physics Division, Rudjer Bošković Institute, P.O.Box 180, 10002 Zagreb, Croatia
}

(Dated: October 26, 2018)

\begin{abstract}
The recent introduction of a deformed non-minimal version of the noncommutative Standard Model in the enveloping-algebra approach, having a one-loop renormalisable gauge sector involving a higher order gauge term, motivates us to consider the possibility of extending the fermion sector with additional deformations, i.e. higher order fermionic terms. Since the renormalisability properties of the fermion sector of the model are not yet fully known, we work with an effective fermion lagrangian which includes noncommutative higher order terms involving a contraction with the noncommutative $\theta$ tensor aside from the star products, so that these terms annihilate in the commutative limit. Some of these terms violate CPT in the weak sector, and some violate CP in the strong and hypercharge sectors. We apply this framework to the reevaluation of the decay rates of quarkonia $\left(\bar{q} q_{1}=J / \psi, \Upsilon\right)$ into two photons. These decays, which are forbidden in the ordinary Standard Model, had been previously studied as possible signals for noncommutativity, but not in the framework of the better behaved deformed non-minimal version of the noncommutative Standard Model. Weak CPT or strong-hypercharge $\mathrm{CP}$ violating interactions do not contribute to the result. If the parameters of the model take natural values, for the vast majority of configurations the resulting branching ratios are enhanced with respect to their values in the minimal version of the noncommutative Standard Model. Also for more than half of the parameter space, the rates are larger than the maximal rates that were calculated in the undeformed version of the non-minimal noncommutative Standard Model. Tuning the dimensionless parameters the predicted branching ratios can fall within the current experimental bounds.
\end{abstract}

PACS numbers: 11.10.Nx, 12.38.-t, 12.39Dc, 12.39.-x, 14.20-c

\section{INTRODUCTION}

The Standard Model (SM) of particle physics and the theory of gravity describe very well, as far as we know today, all physical phenomena from cosmological processes to the properties of subnuclear structures. Nevertheless, at extreme energies and/or very short distances -at the Planck scale- this theories fail to be compatible, which motivates the study of modified or alternative space-time structures that could help to solve the above mentioned difficulties or at least shed some light on them. These modified space-time structures arise in such settings as the quantised coordinates in string theory or in the general framework of deformation quantisation. The idea of noncommutative (NC) space-time, which can be realised in both of the above settings, has recently found more and more interest. In this paper we deal with noncommutative theories defined by means of the enveloping algebra approach, which allows to define gauge theories with arbitrary gauge groups, in particular that of the Standard Model. The research on these theories so far has successfully dealt with some theoretical and also phenomenological aspects, which might allow the confrontation of the theory with experiments.

On the theoretical side, in the enveloping algebra approach developed in [1] following the ideas of the seminal paper [2], we can emphasise the construction of noncommutative minimal and non-minimal versions of the Standard Model (mNCSM and nmNCSM) [3-5], and GUTS [6]. Of particular interest is the deformed version of the nmNCSM with renormalisable pure gauge interactions discussed in refs. [7, 8], which involves an extension of the pure noncommutative gauge lagrangian $\widehat{F} \star \widehat{F}$ with a deformation or higher order term. These noncommutative extensions of the SM are anomaly free $[9,10]$. In contrast with the gauge sector, the renormalisability of the fermion sector has not yet been completely addressed, despite some encouraging partial results [11]. Nevertheless, the results in the pure gauge sector motivate to consider the possible effects of introducing higher order fermionic terms; in this paper we will initiate the study of an extended fermion sector by introducing a general class of the fermionic deformations in the noncommutative action of the nmNCSM and obtaining some of the corresponding Feynman rules.

On the other hand, on the phenomenological side, it is known that noncommutative field theories can predict nonzero rates for processes which are forbidden in the Standard Model due to the Lorentz invariance and Bose symmetry (Landau-Pomeranchuk-Yang or LPY theorem). These new effects follow from the violation of Lorentz invariance in the presence of noncommutativity, and the observation of these forbidden decays could be taken as a signal for it. Some of the SM forbidden processes that have been studied, at the level of tree diagrams, include $Z \rightarrow \gamma \gamma / g g$ [4]; 
$J / \Psi, \Upsilon \rightarrow \gamma \gamma[12]$ and $K \rightarrow \pi \gamma$ [13]. For other phenomenological studies, including limits on the noncommutative scale $\Lambda_{\mathrm{NC}}$, see [14]. The recent introduction of the deformed nmNCSM calls for a reevaluation of the results, which so far has only been done for the $Z \rightarrow \gamma \gamma$ process [15]. In this paper we will apply our framework of a deformed fermionic sector to study the quarkonia decays into two photons $J / \Psi, \Upsilon \rightarrow \gamma \gamma$. There are other processes involving quarkonia in which noncommutativity induces rare decays. An example is the decay into two photons of quarkonia polarised in some direction, say the $x^{3}$ axis. In such polarised rate the contributions proportional to the third components $\left(E_{\theta}^{3}\right)^{2}$ and $\left(B_{\theta}^{3}\right)^{2}$ should be enhanced by a large factor, similarly as in the $Z \rightarrow \gamma \gamma$ case [15]. In addition, one could study decays of quarkonia in other gauge bosons. In general, we expect that any quark-antiquark state with the same quantum numbers as those of $J / \Psi$ and $\Upsilon\left(I^{G}\left(J^{P C}\right)=0^{-}\left(1^{--}\right)\right)$will decay into two gauge bosons through noncommutative interactions. We restrict ourselves to quarkonia decays into two photons because their detection could in principle be achieved within very high-resolution calorimeters by applying more stringent conditions in the selection of the photon candidates when searching for $\gamma \gamma$ events. We could also have decays into a pair of gluons, but these would hadronise into hadron jets, whose detection would be much more problematic due to a lack of localisation of the jets or interference with other signals. Of course there could be also decays into two $\mathrm{Z}$ bosons, but, on the one hand, they would not be produced for quarkonia at rest due to the heaviness of the $\mathrm{Z}$ bosons, and, on the other, these would rapidly decay into other particles.

The paper is organised as follows. First, we give some more detailed fundamental and phenomenological motivations for our work, and we introduce the theoretical framework. Next we introduce the action of the renormalisable pure gauge sector of deformed nmNCSM, after which we deal with the matter sector, extending the fermion action with higher order deformations and examining their C,P,T transformation properties. Following this, we study whether field redefinitions in the fermion sector can help to get rid of some of the extra terms introduced. Finally we give the Feynman rules relevant to the calculation of the quarkonia decay rates and we present our results, which are then discussed.

\section{MOTIVATION AND FRAMEWORK}

Our main goal in this paper is, following the recent introduction of the deformed version of the gauge sector of the noncommutative Standard Model (NCSM), to consistently define the action of an extended fermion sector of the nmNCSM from an effective-theory point of view, by adding higher order terms, and derive relevant Feynman rules. This is certainly important for future investigations about the renormalisability properties of entire NCSM, which are interesting in their own right and could introduce constraints on our "effective" deformed fermion sector. The second goal of this paper is to apply this framework to the calculation of the decay rates of the simplest processes which are forbidden in the ordinary SM, that is the C symmetry violating decay of quarkonia into two photons, $\left(\bar{q} q_{1}=J / \psi, \Upsilon\right)$. These processes are of theoretical interest because their tree-level contributions come from two different types of diagrams, these being s-channel gauge bosons exchanges -involving interactions from the pure gauge sector of the NCSM- and t-channel quark exchanges -involving interactions from the fermion sector. Of course, it should not be forgotten that this proposed processes are also important because they violate the LPY theorem that holds in the ordinary SM, and thus their hypothetical detection could be taken as a possible signal of noncommutativity.

The first construction of the NCSM was undertaken in ref. [3], where it was already noted that there was an ambiguity in the choice of traces for the gauge kinetic terms, leading to a minimal version (with traces taken in the adjoint representation), and a non-minimal version including traces over the representations of all the massive particle multiplets charged under any of the gauge groups [4]. The interaction vertices and Feynman rules of these models were further analysed in refs. [5]. As was said, some of the processes studied in this framework include $Z \rightarrow \gamma \gamma / g g$ $[4,15] ; J / \Psi, \Upsilon \rightarrow \gamma \gamma[12], K \rightarrow \pi \gamma[13]$ and $\gamma_{p l a s m o n} \rightarrow \bar{\nu} \nu[16]$.

At one loop order, the investigations so far were concerned with renormalisability properties [7, 8, 15]; remarkably, it was found that the pure gauge interactions of the nmNCSM could be rendered one-loop renormalisable at first order in the noncommutativity parameters $\theta^{\mu \nu}$ by adding an extra deformation term to the lagrangian involving only gauge fields and their derivatives contracted with one $\theta^{\mu \nu}$. This term was introduced at the level of the noncommutative action $[8,15]$, yielding an extended version of the nmNCSM [7]. This result was reached by considering only gauge field contributions to the loop integrals. When matter fields are included, the results of ref. [17] show that, when computing the matter contributions to the one-loop diagrams with external gauge fields in a generic noncommutative gauge theory with Dirac fermions or complex scalars, the divergences can be absorbed in the bare lagrangian whenever the representations of the matter fields are included in the choice of representations for the traces in the pure gauge terms of the action.

This result motivates the following comments. First, it suggests that there is virtually no hope of getting renormal- 
isability of the gauge sector in the mNCSM case when Dirac fermions run in the loops, since the model only involves traces in the adjoint representation of the gauge fields. However, in the pure gauge sector of the nmNCSM $[4,5]$ the traces include the representations of the matter fields, so that one could say that it is likely that the nmNCSM gauge sector will still be renormalisable if one includes the effects of the matter fields in the loops. This motivates even more to favour the use of the nmNCSM, and in particular its deformed version, over the mNCSM.

Regarding the renormalisability properties of the nmNCSM, we would like to make some clarifying observation. The NC SU(N) pure gauge theory [8] showed renormalisability for two choices of the free deformation parameter $a$, associated with the higher order term, $S_{g}^{H}(a)$, that is present in the deformed nmNCSM: $a=1$ and $a=3$. However, this result cannot be extrapolated directly to the NCSM, because the gauge fields mix after the SW map and it is not a sum of $\mathrm{NC} \mathrm{SU}(\mathrm{N})$ theories. In fact, the gauge sector of the nmNCSM is only renormalisable for $a=3$, even more, the one-loop quantum corrections are finite for $a=3$.

The above arguments clearly favours the nmNCSM over the mNCSM if one wants to have a better behaved theory, where the quantum corrections are more under control even when working in an effective theory approach. In particular, predictions of the theory involving pure gauge boson interactions will be more robust under changes of scale.

All these new results call for a reevaluation, in the framework of the deformed nmNCSM, of the possible signals for noncommutativity that were commented upon before. As was said before, this has already been done in ref. [15] for the $Z \rightarrow \gamma \gamma$ decay. In this paper we will focus on the disintegration $J / \Psi, \Upsilon \rightarrow \gamma \gamma$. Now, since the renormalisability properties of the fermion sector of deformed nmNCSM are essentially unknown, despite promising results for some diagrams involving chiral fermions [11], and since the results for the pure gauge sector make us expect that a renormalisable fermion sector could only be achieved by adding deformed fermionic contributions to the action, it makes sense to treat this sector effectively and consider, aside from the fermion lagrangian employed in both mNCSM and nmNCSM, higher order contributions involving contractions with $\theta^{\mu \nu}$ outside the star product and compatible with the noncommutative gauge symmetry. We will demand that these deformed fermionic contributions do not introduce further violations of parity than those coming from the ordinary $S U(2)_{L}$ gauge fields; also, we will require that they do not alter the tree level fermion 2-point function. This modification of the propagator could be achieved, for example, with terms like $i \overline{\hat{\psi}} \theta^{\alpha \beta} \gamma_{\beta}\left\{\hat{D}^{2}, \hat{D}_{\alpha}\right\} \star \hat{\psi}$. The problem is that, when computing S-matrix elements, one ordinarily uses the LSZ formalism, which is constructed by using Lorentz invariance and implies that the S-matrix elements are obtained by identifying the poles in momentum space of some Green functions. This happen at points satisfying the Lorentz invariant constraint $p^{2}=m^{2}$ for some $m$. In noncommutative spacetime Lorentz invariance is broken, which means that the poles of the Green functions need not satisfy the constraint $p^{2}=m^{2}$; a $\theta$-dependence may appear and then one should be more careful when computing S-matrix elements. An alternative definition is needed, perhaps along the lines of the work done in ref. [18] for noncommutative theories formulated without SW maps. Nevertheless, if the tree-level propagator still has a pole of the ordinary type, we expect that the usual way of deriving matrix elements will be valid to some approximation. Here we pretend to compute S-matrix elements at tree level only.

\section{PURE GAUGE SECTOR}

\section{Renormalisable pure gauge sector action}

The pure gauge part of the deformed nmNCSM is given by [15]:

$$
\begin{aligned}
S_{g} & =S_{g}^{\min }+S_{g}^{H}(a), \\
S_{g}^{\text {min }} & =-\frac{1}{2} \operatorname{Tr} \int d^{4} x \widehat{F}_{\mu \nu} \star \widehat{F}^{\mu \nu}, \quad S_{g}^{H}(a)=\frac{a-1}{4} \operatorname{Tr} \int d^{4} x h \theta^{\mu \nu} \star \widehat{F}_{\mu \nu} \star \widehat{F}_{\rho \sigma} \star \widehat{F}^{\rho \sigma},
\end{aligned}
$$

where the trace $\operatorname{Tr}$ is taken over all the particle representations. As usual, the Moyal-Weyl $\star$-product is given by $f \star g=f\left(\exp \frac{i}{2} h \theta^{\mu \nu} \overleftarrow{\partial}_{\mu} \vec{\partial}_{\nu}\right) g$, which implements the space-time noncommutativity as $x^{\mu} \star x^{\nu}-x^{\nu} \star x^{\mu}=i h \theta^{\mu \nu}$ The noncommutative deformation parameter $h=1 / \Lambda_{\mathrm{NC}}^{2}$ sets the noncommutative scale. The noncommutative field strength $\hat{F}^{\mu \nu}$ depends on the enveloping-algebra valued noncommutative gauge field $\hat{V}_{\mu}$ as

$$
\widehat{F}_{\mu \nu}(x)=\partial_{\mu} \widehat{V}_{\nu}-\partial_{\nu} \widehat{V}_{\mu}-i\left[\widehat{V}_{\mu}, \widehat{V}_{\nu}\right]
$$


and in turn, $\hat{V}_{\mu}$ depends on the ordinary gauge bosons through the Seiberg-Witten map

$$
\begin{aligned}
\widehat{V}_{\mu}(x) & =V_{\mu}(x)-\frac{h}{4} \theta^{\sigma \rho}\left\{V_{\sigma}(x), \partial_{\rho} V_{\mu}(x)+F_{\rho \mu}(x)\right\}+O\left(h^{2}\right), \\
V_{\mu}(x) & =g^{\prime} \mathcal{A}_{\mu}(x) Y+g \sum_{a=1}^{3} B_{\mu, a}(x) T_{L}^{a}+g_{s} \sum_{b=1}^{8} G_{\mu, b}(x) T_{S}^{b},
\end{aligned}
$$

where $V_{\mu}(x)$ is the Standard Model gauge potential taking values in the Lie algebra of $S U(3)_{C} \times S U(2)_{L} \times U(1)_{Y}$. The pure gauge action defined by eq. (1), after expanding to order $h$ with the SW map of eq. (3) leads to

$$
S_{g}=\operatorname{Tr} \int \mathrm{d}^{4} x\left(-\frac{1}{2} F_{\mu \nu} F^{\mu \nu}+h \theta^{\mu \nu}\left(\frac{a}{4} F_{\mu \nu} F_{\rho \sigma}-F_{\mu \rho} F_{\nu \sigma}\right) F^{\rho \sigma}\right) .
$$

After taking traces in the above action over all massive particle representations with different quantum numbers that appear in the total lagrangian of the model with covariant derivatives acting on them, the gauge action (5) produces triple neutral gauge boson interactions $[4,5]$ which are not present in the mNCSM; in this paper we are interested in the $\gamma \gamma \gamma$ and $Z \gamma \gamma$ couplings, which arise from the following terms in the lagrangian [15]

$$
\begin{aligned}
\mathcal{L}_{\gamma \gamma \gamma}^{\mathrm{nmNCSM}}(a) & =\frac{e}{4} \sin 2 \theta_{W} K_{\gamma \gamma \gamma} h \theta^{\rho \sigma} A^{\mu \nu}\left(a A_{\mu \nu} A_{\rho \sigma}-4 A_{\mu \rho} A_{\nu \sigma}\right), \\
\mathcal{L}_{Z \gamma \gamma}^{\mathrm{nmNCSM}}(a) & =\frac{e}{4} \sin 2 \theta_{W} K_{Z \gamma \gamma} h \theta^{\rho \sigma}\left[2 Z^{\mu \nu}\left(2 A_{\mu \rho} A_{\nu \sigma}-a A_{\mu \nu} A_{\rho \sigma}\right)+8 Z_{\mu \rho} A^{\mu \nu} A_{\nu \sigma}-a Z_{\rho \sigma} A_{\mu \nu} A^{\mu \nu}\right] .
\end{aligned}
$$

See fig. 1 for details on the allowed values for the constants $K_{\gamma \gamma \gamma}$ and $K_{Z \gamma \gamma}$ and their dependence on other parameters of the model [4]. The remarkable result of refs. [7] is that the gauge action (5) is one-loop renormalisable up to order $h$ for $a=3$, with the $O(h)$ quantum corrections being finite. Thus we will be mainly interested in the $a=3$ case.

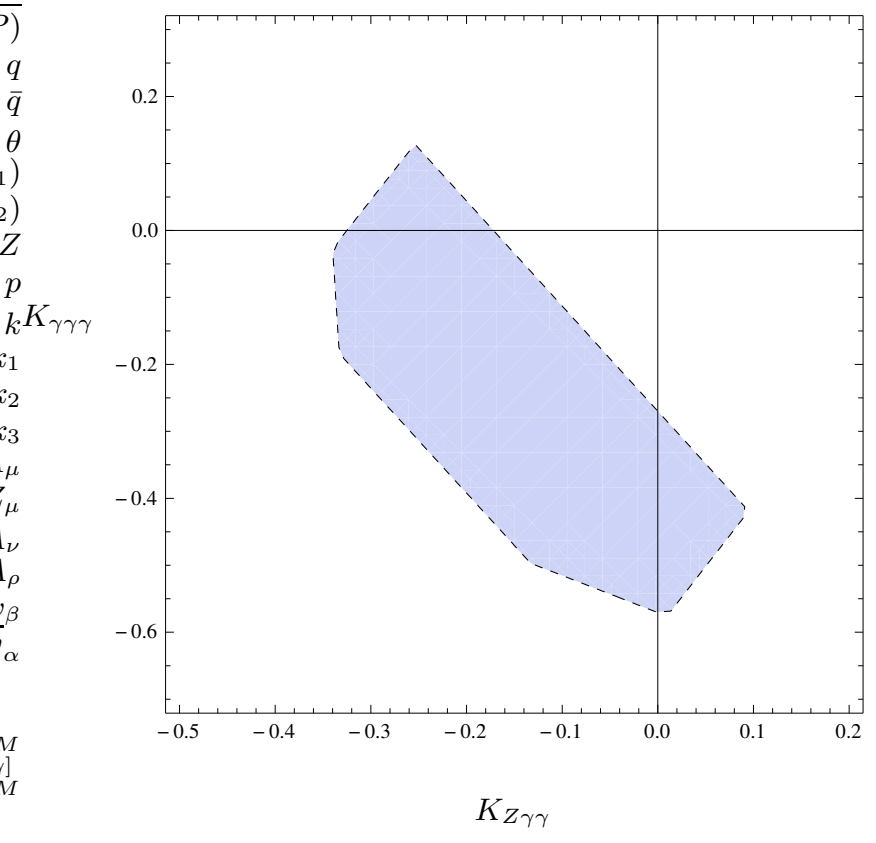

FIG. 1: Allowed values for the couplings $K_{Z \gamma \gamma}$ and $K_{\gamma \gamma \gamma}$ in the nmNCSM.

\section{MATTER SECTOR}

\section{Minimal fermion action}

Next we turn our attention to the fermion sector. In both the mNCSM and nmNCSM models, the chosen minimal fermion action was

$$
S_{\psi}^{\min }=\int d^{4} x \widehat{\widehat{\psi}} \star i \hat{D} \star \widehat{\psi}+\hat{S}_{\text {Yukawa }}, \quad \hat{D}_{\mu}=\partial_{\mu}-i \hat{V}_{\mu} \star
$$


which depends on the ordinary fields $V_{\mu}$ and $\psi$ through the SW map of eq. (3) and the fermion SW map

$$
\widehat{\psi}=\psi-\frac{h}{2} \theta^{\sigma \rho}\left(V_{\sigma} \partial_{\rho}-\frac{i}{4}\left[V_{\sigma}, V_{\rho}\right]\right) \psi+O\left(h^{2}\right) .
$$

The Yukawa sector in the NCSM, when expanded in terms of ordinary fields, involves quite complicated interactions [5]. However, if we consider the noncommutative contributions to the fermion field interactions in the QED sector only, (as turns out to be sufficient for our purpose of obtaining rate for the quarkonia decay into two photons), we can use, as noted in [5] and used in [12], the simplified fermion Lagrangian

$$
\begin{aligned}
S_{\psi, A}^{\min } & \sim \int d^{4} x \overline{\widehat{\psi}} \star\left(i \hat{D}^{A}-m_{f}\right) \star \widehat{\psi}=S_{\psi, A}^{S M}+S_{\psi, A}^{\theta}+O\left(h^{2}\right), \\
S_{\psi, A}^{S M} & =\int d^{4} x \bar{\psi}\left(i D^{A}-m_{f}\right) \psi, \quad S_{\psi, A}^{\theta}=-\frac{e h}{4} \int d^{4} x \bar{\psi} A_{\mu \nu}\left(i \theta^{\mu \nu \rho} D_{\rho}^{A}-m_{f} \theta^{\mu \nu}\right) \psi,
\end{aligned}
$$

where $D_{\mu}^{A}=\partial_{\mu}-i e A_{\mu}$ and

$$
\theta^{\mu \nu \rho}=\theta^{\mu \nu} \gamma^{\rho}+\theta^{\nu \rho} \gamma^{\mu}+\theta^{\rho \mu} \gamma^{\nu}
$$

\section{Deformed fermion action}

Motivated by the unknown renormalisability properties of the $\mathrm{NC}$ fermion sector, and since in the case of the gauge sector the addition of the higher order term dependent on the free deformation parameter $a$ made the model surprisingly well-behaved, we will consider adding deformation terms to the minimal fermion lagrangian (9), either to treat it in an effective theory approach or to prepare the grounds for future investigations of renormalisability. Thus, we set to find all the possible deformations contributing to the fermion action, and satisfying the following conditions:

(a) They are real and include two fermions fields,

(b) are invariant under noncommutative gauge transformations and thus involve star products, noncommutative gauge covariant derivatives and noncommutative field strengths,

(c) involve a contraction with a $\theta^{\mu \nu}$ tensor outside the star product — as in the $a$-dependent term in eq. (1) -,

(d) they do not alter the tree-level 2-point function of fermion propagator,

(e) they only generate P-symmetry violating contributions coming from the ordinary weak $\mathrm{SU}(2)$ gauge fields,

(f) include zero or positive powers of some mass parameter.

In order to write down all the possible terms satisfying the conditions above, we have to consider a basis of matrices in spinor space. Since the $\gamma$ matrices are constrained by the identity $\left\{\gamma^{\mu}, \gamma^{\nu}\right\}=2 g^{\mu \nu}$, we can consider a basis formed by antisymmetrised products of $\gamma$ matrices: $\left\{I, \gamma^{\mu}, \sigma^{\mu \nu}, \gamma^{\mu \nu \rho}, \gamma^{5}\right\}$, where we are using the following definitions:

$$
\begin{aligned}
& \sigma^{\mu \nu}=\frac{1}{2}\left(\gamma^{\mu} \gamma^{\nu}-\gamma^{\nu} \gamma^{\mu}\right), \\
& \gamma^{\mu \nu \rho}=\frac{1}{6}\left(\gamma^{\mu} \gamma^{\nu} \gamma^{\rho}+\gamma^{\rho} \gamma^{\mu} \gamma^{\nu}+\gamma^{\nu} \gamma^{\rho} \gamma^{\mu}-\gamma^{\nu} \gamma^{\mu} \gamma^{\rho}-\gamma^{\rho} \gamma^{\nu} \gamma^{\mu}-\gamma^{\mu} \gamma^{\rho} \gamma^{\nu}\right), \\
& \gamma^{\mu} \gamma^{\nu} \gamma^{\rho}=g^{\mu \nu} \gamma^{\rho}-g^{\mu \rho} \gamma^{\nu}+g^{\nu \rho} \gamma^{\mu}-i \epsilon^{\mu \nu \rho \sigma} \gamma^{5} \gamma_{\sigma}, \\
& \gamma^{5}=-\frac{i}{4 !} \epsilon_{\mu \nu \rho \sigma} \gamma^{\mu} \gamma^{\nu} \gamma^{\rho} \gamma^{\sigma} .
\end{aligned}
$$

With this in mind, it can be seen that the terms satisfying the conditions stated above are given by sums of integrals of the following monomials $t_{i}$ multiplied by real coefficients:

$$
\begin{aligned}
& t_{1}=h \theta^{\alpha \beta} \overline{\widehat{\psi}} \star \gamma^{\mu}\left(\hat{\mathcal{D}}_{\mu} \hat{F}_{\alpha \beta}\right) \star \widehat{\psi}, \quad t_{2}=h \theta^{\alpha \beta} \overline{\bar{\psi}} \star \gamma_{\beta}\left(\hat{\mathcal{D}}^{\mu} \hat{F}_{\mu \alpha}\right) \star \widehat{\psi}, \\
& t_{3}=i h \theta^{\alpha \beta} \overline{\bar{\psi}} \star \gamma^{\rho}\left(2 \hat{F}_{\alpha \beta} \star \hat{D}_{\rho}+\left(\hat{\mathcal{D}}_{\rho} \hat{F}_{\alpha \beta}\right)\right) \star \widehat{\psi}, \quad t_{4}=i h \theta^{\alpha \beta} \overline{\bar{\psi}} \star \gamma^{\rho}\left(2 \hat{F}_{\beta \rho} \star \hat{D}_{\alpha}+\left(\hat{\mathcal{D}}_{\alpha} \hat{F}_{\beta \rho}\right)\right) \star \widehat{\psi}, \\
& t_{5}=i h \theta^{\alpha \beta} \overline{\widehat{\psi}} \star \gamma_{\beta}\left(2 \hat{F}_{\mu \alpha} \star \hat{D}^{\mu}+\left(\hat{\mathcal{D}}^{\mu} \hat{F}_{\mu \alpha}\right)\right) \star \widehat{\psi}, \quad t_{6}=i h \theta^{\alpha \beta} \overline{\widehat{\psi}} \star \gamma_{\alpha \beta}{ }^{\rho}\left(\hat{\mathcal{D}}^{\mu} \hat{F}_{\mu \rho}\right) \star \widehat{\psi}, \\
& t_{7}=i h \theta^{\alpha \beta} \overline{\widehat{\psi}} \star \gamma_{\beta}{ }^{\mu \nu}\left(\hat{\mathcal{D}}_{\alpha} \hat{F}_{\mu \nu}\right) \star \widehat{\psi}, \quad t_{8}=h \theta^{\alpha \beta} \overline{\widehat{\psi}} \star \gamma_{\alpha \beta}{ }^{\rho}\left(2 \hat{F}_{\mu \rho} \star \hat{D}^{\mu}+\left(\hat{\mathcal{D}}^{\mu} \hat{F}_{\mu \rho}\right)\right) \star \widehat{\psi}, \\
& t_{9}=h \theta^{\alpha \beta} \overline{\widehat{\psi}} \star \gamma_{\beta}{ }^{\mu \nu}\left(2 \hat{F}_{\mu \nu} \star \hat{D}_{\alpha}+\left(\hat{\mathcal{D}}_{\alpha} \hat{F}_{\mu \nu}\right)\right) \star \widehat{\psi}, \quad t_{10}=h \theta^{\alpha \beta} \overline{\widehat{\psi}} \gamma_{\beta}{ }^{\mu \nu}\left(2 \hat{F}_{\nu \alpha} \star \hat{D}_{\mu}+\left(\hat{\mathcal{D}}_{\mu} \hat{F}_{\nu \alpha}\right)\right) \star \widehat{\psi}, \\
& t_{11}=m h \theta^{\alpha \beta} \overline{\widehat{\psi}} \star \hat{F}_{\alpha \beta} \star \widehat{\psi} \\
& t_{13}=i m h \tilde{\theta}^{\alpha \beta} \overline{\hat{\psi}} \star \gamma^{5} \hat{F}_{\alpha \beta} \star \widehat{\psi},
\end{aligned}
$$


with $\tilde{\theta}^{\alpha \beta} \equiv \frac{1}{2} \epsilon^{\alpha \beta \rho \sigma} \theta_{\rho \sigma}$, and $\hat{D}_{\mu}=\partial_{\mu}-i \hat{V}_{\mu} \star, \quad \hat{\mathcal{D}}_{\mu}=\partial_{\mu}-i\left[\hat{V}_{\mu}^{\star} \stackrel{\text {. }}{]}\right]$. Note that we can identify the mass parameter $m$ with any of the fermion masses $m_{f}$; this means no loss of generalisation because in principle we will be adding these mass-dependent terms to the action multiplied by arbitrary dimensionless coefficients.

In order to check the C,P,T transformations of the above terms, we consider the following:

- $\theta^{\mu \nu}$ transforms under discrete space-time symmetries as a U(1) field strength $F^{\mu \nu}[6]$.

- The ordinary fields are the ones that define the theory, so that we have to deal with their C,P,T transformation properties. However, the analysis can be simplified because of the fact that the SW maps that we use are such that the C,P,T transformations of the noncommutative fields are equal to their commutative counterparts [6]. However, there is a subtlety since the noncommutative vector field in the matter representations, given the expansion of eq. (4), includes some chiral projectors that come together with the weak gauge fields $B_{\mu, a}(x)$.

Taking this into account, it can be seen that the above terms are $\mathrm{P}$ invariant save for the contributions involving the ordinary $\mathrm{SU}(2)$ gauge fields, as was required from the start. Moreover, all terms in eqs. (12) are CPT invariant except for $t_{11}, t_{12}, t_{13}$. In the terms $t_{11}, t_{12}, t_{13}$ the $\mathrm{CPT}$ violating contributions come exclusively from the chiral projectors of the weak gauge fields. However, in the strong and hypercharge sectors the contributions from all terms in (12) remain $\mathrm{CPT}$ invariant. Interestingly, the terms $t_{1}, t_{2}, t_{8}, t_{9}, t_{10}, t_{12}$ originate both $\mathrm{C}$ and $\mathrm{T}$ violations in the strong and hypercharge sectors, with CT conserved in these sectors. All other violations of C, P or T come from the weak fields exclusively. We will not worry about these violations of discrete symmetries and will proceed considering all of the terms in eq. (12). Thus, we will consider an additional piece of the action given by a sum of the above terms,

$$
S_{\psi}^{H}\left(x_{i}\right)=\int d^{4} x \sum_{i} x_{i} t_{i}, \quad x_{i} \in \mathbb{R},
$$

where $x_{i}$ 's are the fermion sector free deformation parameters, to be constrained via considerations of renormalisability or by phenomenology.

Before plunging into the computation of the Feynman rules and their phenomenological application, we would like to analyse whether any of the terms in eq. (12) can be reabsorbed by means of field redefinitions. This is interesting for two reasons:

- Possible future investigations about renormalisability would require to take into account the effects of field redefinitions in the action, which we will compute here in the fermion sector.

- Field redefinitions should not affect S-matrix elements, and since we aim to calculate quarkonia decay rates it is desirable to eliminate as many of the $x_{i}$ parameters in eq. (13) as possible.

To proceed, we consider the most general field redefinitions of order $\theta$ of the commutative gauge and fermion fields, involving zero or positive powers of the mass parameter and not affecting the tree-level propagator, and compute the associated change in the action. We parameterise the most general field redefinitions up to order $\theta$ as

$$
\delta v_{\mu}=\sum y_{i} \delta^{i} v_{\mu}, \quad \delta \psi=\sum z_{i} \delta^{i} \psi
$$

where $y_{i}$ are real, $z_{i}$ complex, and $\delta^{i} v_{\mu}$ and $\delta^{i} \psi$ are given next

$$
\begin{aligned}
& \delta^{1} v_{\mu}=\theta^{\alpha \beta} \mathcal{D}_{\mu} F_{\alpha \beta}, \quad \delta^{2} v_{\mu}=\theta_{\mu}{ }^{\alpha} \mathcal{D}^{\nu} F_{\nu \alpha}, \\
& \delta^{1} \psi=\theta^{\alpha \beta} F_{\alpha \beta} \psi, \quad \delta^{2} \psi=\theta^{\alpha \beta} \sigma_{\beta}{ }^{\rho} F_{\alpha \rho} \psi, \quad \delta^{3} \psi=\tilde{\theta}^{\alpha \beta} \gamma^{5} F_{\alpha \beta} \psi .
\end{aligned}
$$

Since the field redefinitions are of order $h$, to see their effect on the action to this same order we only need to compute the variation of the $O\left(h^{0}\right)$ action, which for fermions and vector fields is just

$$
S_{v, \psi}^{S M}=S_{g}^{S M}+\int d^{4} x \bar{\psi}\left(i \not D-m_{f}\right) \psi+S_{\text {Yukawa }} ;
$$

we will ignore the Yukawa couplings since they do not contribute to the tree-level quarkonia decay amplitudes that we want to compute. The change of the total action turns out to be

$$
\delta\left(S_{g}^{S M}+\int d^{4} x \bar{\psi}\left(\not \not D-m_{f}\right) \psi\right)=\int d^{4} x \sum_{i}\left(y_{i} \delta^{y_{i}} \mathcal{L}+\operatorname{Re} z_{i} \delta^{z_{i}, R} \mathcal{L}+\operatorname{Im} z_{i} \delta^{z_{i}, I} \mathcal{L}\right)+O\left(h^{2}\right),
$$


with

$$
\begin{aligned}
& \delta^{y_{1}} \mathcal{L}=t_{1}, \quad \delta^{y_{2}} \mathcal{L}=-t_{2}, \quad \delta^{z_{1}, R} \mathcal{L}=t_{3}-2 t_{11}, \\
& \delta^{z_{1}, I} \mathcal{L}=-t_{1}, \quad \delta^{z_{2}, R} \mathcal{L}=-t_{4}+t_{5}-\frac{1}{2} t_{7}, \\
& \delta^{z_{3}, R} \mathcal{L}=-t_{8}-t_{9}, \quad \delta^{z_{3}, I} \mathcal{L}=-t_{6}-t_{7}-2 t_{13},
\end{aligned}
$$

where the $t_{i}$ 's are given in eq. (12). In the language of eq. (13), the above result is equivalent to the following change in the action $S_{\psi}^{H}\left(x_{i}\right)$ :

$$
S_{\psi}^{H}\left(x_{i}\right) \longrightarrow S_{\psi}^{H}\left(x_{i}+\delta x_{i}\right)
$$

with

$$
\begin{aligned}
& \delta x_{1}=y_{1}-\operatorname{Im} z_{1}-\frac{1}{2} \operatorname{Im} z_{2}, \\
& \delta x_{2}=-y_{2}-\operatorname{Im} z_{2}, \\
& \delta x_{3}=\operatorname{Re} z_{1}, \\
& \delta x_{4}=-\operatorname{Re} z_{2}, \\
& \delta x_{5}=\operatorname{Re} z_{2}, \\
& \delta x_{6}=-\operatorname{Im} z_{3}, \\
& \delta x_{7}=-\frac{1}{2} \operatorname{Re} z_{2}-\operatorname{Im} z_{3}, \\
& \delta x_{8}=-\operatorname{Re} z_{3}, \\
& \delta x_{9}=-\operatorname{Re} z_{3}, \\
& \delta x_{10}=-\operatorname{Im} z_{2} \text {, } \\
& \delta x_{11}=-2 \operatorname{Re} z_{1}, \\
& \delta x_{12}=-2 \operatorname{Im} z_{2}, \\
& \delta x_{13}=-2 \operatorname{Im} z_{3} \text {. }
\end{aligned}
$$

In order to check whether the field redefinitions above can be used to eliminate some of the terms $t_{i}$ of the action in eq. (13), we have to examine the system of equations that follows

$$
\delta x_{i}\left[y_{j}, \operatorname{Re} z_{j}, \operatorname{Im} z_{j}\right]=-x_{i}
$$

where $x_{j}$ are to be treated as fixed and $y_{i}, \operatorname{Re} z_{i}, \operatorname{Im} z_{i}$ as the unknown variables. Now, the $13 \times 8$ matrix associated to the previous linear system of equations can be seen to be of rank 7 . This means that at most 7 of the $t_{i}$ terms in the action can be eliminated with adequate field redefinitions, with 6 terms remaining. It is easily seen that a viable choice of 6 terms that survive the field redefinitions is given by $t_{4}, t_{5}, t_{6}, t_{8}, t_{10}, t_{11}-$ note that, as was commented before, $t_{11}$ violates $\mathrm{CPT}$ in the weak sector and $t_{8}, t_{10}$ violate $\mathrm{C}$ and $\mathrm{T}$ in the strong and hypercharge sectors.

\section{FEYNMAN RULES TO COMPUTE S-MATRIX ELEMENTS}

From the discussions above it follows that, in order to compute the amplitudes of the desired physical processes, the relevant pieces of the action are those given by the ordinary Standard Model fermion-photon and fermion-Z boson interactions, plus the following noncommutative $\theta$-dependent interactions: the three boson interactions of eq. (6), the fermion-photon interaction in eq. (9), and the contribution to the action of the terms of eq. (13) surviving the fermion field redefinitions, i.e.,

$$
S_{\psi}^{H}\left(x_{i}\right)=\int d^{4} x\left[x_{4} t_{4}+x_{5} t_{5}+x_{6} t_{6}+x_{8} t_{8}+x_{10} t_{10}+x_{11} t_{11}\right] .
$$

The noncommutative interactions originate, among other vertices, a triple neutral gauge boson vertex, a 2 fermionphoton vertex and a 2 fermion-2 boson vertex. From the modified gauge and fermion actions, $(1,5,6,9,22)$,

$$
S=S_{g}+S_{\psi}=S_{g}^{m i n}+S_{g}^{H}(a)+S_{\psi}^{m i n}+S_{\psi}^{H}\left(x_{i}\right)+O\left(h^{2}\right),
$$

we obtain the following Feynman rules:



$$
\begin{aligned}
\leftrightarrow & i e e_{q}\left[\gamma^{\mu}-i \frac{h}{2} k_{\nu}\left(\theta_{H}^{\mu \nu \rho} p_{\rho}+\tilde{\theta}_{H}^{\mu \nu \rho} k_{\rho}-\left(1+4 x_{11}\right) m_{f} \theta^{\mu \nu}\right)\right]_{\alpha \beta}, \\
\theta_{H}^{\mu \nu \rho}= & \theta^{\mu \nu \rho}-4 x_{4}\left(\theta^{\rho \mu} \gamma^{\nu}+\theta^{\nu \rho} \gamma^{\mu}\right)-4 x_{5}\left(-g^{\nu \rho} \theta^{\mu \alpha} \gamma_{\alpha}+g^{\mu \rho} \theta^{\nu \alpha} \gamma_{\alpha}\right) \\
& +8 i x_{8} g^{\mu \rho} \theta^{\sigma \eta} \gamma_{\sigma \eta}{ }^{\nu}+8 i x_{10} \theta^{\nu \sigma} \gamma_{\sigma}{ }^{\rho \mu}, \\
\tilde{\theta}_{H}^{\mu \nu \rho}= & \frac{1}{2}\left(\theta_{H}^{\mu \nu \rho}-\theta^{\mu \nu \rho}\right)-4 x_{6} g^{\rho \mu} \theta^{\sigma \eta} \gamma_{\sigma \eta}{ }^{\nu}, \quad e_{q}=\frac{2}{3},-\frac{1}{3}, \text { for quarks, }
\end{aligned}
$$




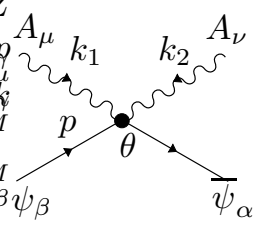

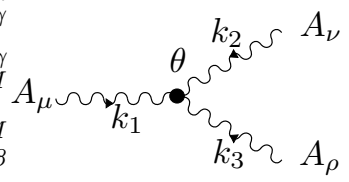

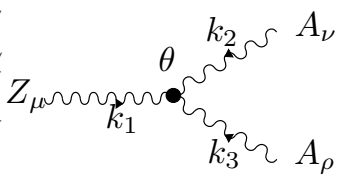

$$
\leftrightarrow-\frac{h}{2} e^{2} e_{q}^{2}\left[\theta_{H}^{\mu \nu \rho}\right]_{\alpha \beta}\left(k_{1}-k_{2}\right)^{\rho},
$$

$$
\leftrightarrow 2 e \sin \left(2 \theta_{W}\right) K_{\gamma \gamma \gamma} h \Theta_{3}^{\mu \nu \rho}\left[a ; k_{1}, k_{2}, k_{3}\right],
$$

$$
\leftrightarrow-2 e \sin \left(2 \theta_{W}\right) K_{Z \gamma \gamma} h \Theta_{3}^{\mu \nu \rho}\left[a ; k_{1}, k_{2}, k_{3}\right] .
$$

Here, $\theta^{\mu \nu \rho}$ was given in eq. (10), while the tensor $\Theta_{3}^{\mu \nu \rho}\left[a ; k_{1}, k_{2}, k_{3}\right]$, is given by [15]:

$$
\begin{aligned}
\Theta_{3}^{\mu \nu \rho}\left[a ; k_{1}, k_{2}, k_{3}\right] & =-\left(k_{1} \theta k_{2}\right)\left[\left(k_{1}-k_{2}\right)^{\rho} g^{\mu \nu}+\left(k_{2}-k_{3}\right)^{\mu} g^{\nu \rho}+\left(k_{3}-k_{1}\right)^{\nu} g^{\rho \mu}\right] \\
& -\theta^{\mu \nu}\left[k_{1}^{\rho}\left(k_{2} k_{3}\right)-k_{2}^{\rho}\left(k_{1} k_{3}\right)\right]-\theta^{\nu \rho}\left[k_{2}^{\mu}\left(k_{3} k_{1}\right)-k_{3}^{\mu}\left(k_{2} k_{1}\right)\right]-\theta^{\rho \mu}\left[k_{3}^{\nu}\left(k_{1} k_{2}\right)-k_{1}^{\nu}\left(k_{3} k_{2}\right)\right] \\
& +\left(\theta k_{2}\right)^{\mu}\left[g^{\nu \rho} k_{3}^{2}-k_{3}^{\nu} k_{3}^{\rho}\right]+\left(\theta k_{3}\right)^{\mu}\left[g^{\nu \rho} k_{2}^{2}-k_{2}^{\nu} k_{2}^{\rho}\right] \\
& +\left(\theta k_{3}\right)^{\nu}\left[g^{\mu \rho} k_{1}^{2}-k_{1}^{\mu} k_{1}^{\rho}\right]+\left(\theta k_{1}\right)^{\nu}\left[g^{\mu \rho} k_{3}^{2}-k_{3}^{\mu} k_{3}^{\rho}\right] \\
& +\left(\theta k_{1}\right)^{\rho}\left[g^{\mu \nu} k_{2}^{2}-k_{2}^{\mu} k_{2}^{\nu}\right]+\left(\theta k_{2}\right)^{\rho}\left[g^{\mu \nu} k_{1}^{2}-k_{1}^{\mu} k_{1}^{\nu}\right] \\
& +(a-1)\left(\left(\theta k_{1}\right)^{\mu}\left[g^{\nu \rho}\left(k_{3} k_{2}\right)-k_{3}^{\nu} k_{2}^{\rho}\right]+\left(\theta k_{2}\right)^{\nu}\left[g^{\mu \rho}\left(k_{3} k_{1}\right)-k_{3}^{\mu} k_{1}^{\rho}\right]+\left(\theta k_{3}\right)^{\rho}\left[g^{\mu \nu}\left(k_{2} k_{1}\right)-k_{2}^{\mu} k_{1}^{\nu}\right]\right) .
\end{aligned}
$$

\section{APPLICATION OF THE PROPOSED FRAMEWORK TO QUARKONIA DECAYS: $\bar{q} q_{1} \rightarrow \gamma \gamma$}

\section{Quarkonia decay amplitudes}

The diagrams that contribute to the quarkonia decay amplitude are shown in figures 2 and 3 . Note that the vertices with fermions including noncommutative effects (with black dots), only involve photons; that's why we may safely use (9). The diagram in fig. 3 also involves a fermion-fermion-Z boson vertex, but it is given by the ordinary SM contribution.

Using the Feynman rules (24-27) obtained from the modified gauge (6) and fermion (23) actions, we have evaluated the diagrams from Figs. 2 and 3, yielding amplitudes that we call $\mathcal{A}_{1}$ and $\mathcal{A}_{2}$, respectively.

As in ref. [12], in order to hadronise the free quarks into the quarkonium bound state, we apply the following prescription for the transition amplitude of the operator $q_{i}^{\alpha} \bar{q}_{j}^{\beta}(q=c, b$ and $i, j$ are colour indices) from the vacuum to the quarkonium state:

$$
\left\langle 0\left|q_{i}^{\alpha} \bar{q}_{j}^{\beta}\right| \bar{q} q_{1}(P)\right\rangle=-\frac{\left|\Psi_{\bar{q} q_{1}}(0)\right|}{\sqrt{12 M}}[(\not P+M) \notin]^{\alpha \beta} \delta_{i j},
$$

where $\left|\Psi_{\bar{q} q}(0)\right|$ represents the quarkonia wave function at the origin defined in [12],

$$
\left|\Psi_{\bar{q} q_{1}}(0)\right|^{2}=\frac{\Gamma\left(\bar{q} q_{1} \rightarrow \ell^{+} \ell^{-}\right) M^{2}}{16 \pi \alpha^{2} e_{q}^{2}} .
$$

We use a collinear approximation for the quarks in the quarkonium state, and we identify the mass of the quarkonium 

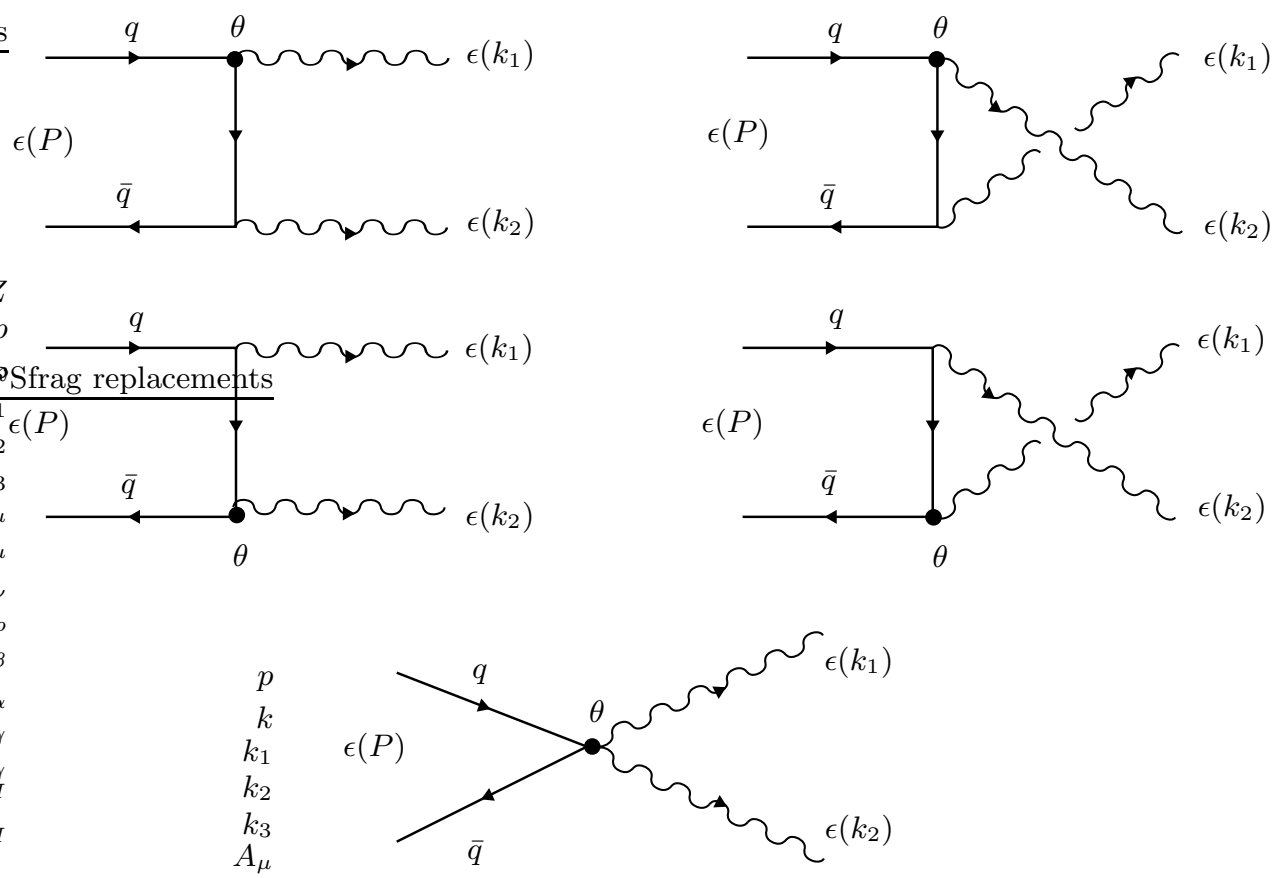

FIG. 2: Contributions to the $\mathcal{A}_{1}\left(\bar{q} q_{1} \rightarrow \gamma \gamma\right)$ amplitude.



FIG. 3: Additional contributions to the $\mathcal{A}_{2}\left(\bar{q} q_{1} \rightarrow \gamma \gamma\right)$ amplitude.

as $M=2 m_{q}$. The resulting amplitudes are shown next:

$$
\begin{aligned}
\mathcal{A}_{1}\left(x_{4}, x_{5}, x_{11}\right) & =i h \pi 4 \sqrt{3 M} \alpha e_{q}^{2}\left|\Psi_{\bar{q} q_{1}}(0)\right| \epsilon_{\mu}\left(k_{1}\right) \epsilon_{\nu}\left(k_{2}\right) \epsilon_{\rho}(P)\left\{( k _ { 2 } - k _ { 1 } ) ^ { \rho } \left[\left(1-4 x_{4}\right)\left(\theta^{\mu \nu}-2 g^{\mu \nu} \frac{\left(k_{1} \theta k_{2}\right)}{M^{2}}\right)\right.\right. \\
& \left.-4 x_{5}\left(\theta^{\mu \nu}-\frac{k_{2}^{\mu}\left(k_{1} \theta\right)^{\nu}-k_{1}^{\nu}\left(k_{2} \theta\right)^{\mu}}{M^{2}}\right)\right]-4 x_{5}(P \theta)^{\rho}\left(g^{\mu \nu}-\frac{2}{M^{2}} k_{2}^{\mu} k_{1}^{\nu}\right) \\
& +\left(1-4 x_{4}-2 x_{5}\right)\left[2 g^{\mu \rho}\left(\left(k_{1} \theta\right)^{\nu}-2 k_{1}^{\nu} \frac{\left(k_{1} \theta k_{2}\right)}{M^{2}}\right)+2 g^{\nu \rho}\left(\left(k_{2} \theta\right)^{\mu}+2 k_{2}^{\mu} \frac{\left(k_{1} \theta k_{2}\right)}{M^{2}}\right)\right] \\
& \left.+\left(4 x_{11}\right)\left[\left(k_{1} \theta\right)^{\mu}\left(g^{\nu \rho}+\frac{k_{1}^{\rho} k_{1}^{\nu}-k_{2}^{\rho} k_{1}^{\nu}}{M^{2}}\right)+\left(k_{2} \theta\right)^{\nu}\left(g^{\mu \rho}+\frac{k_{2}^{\rho} k_{2}^{\mu}-k_{1}^{\rho} k_{2}^{\mu}}{M^{2}}\right)\right]\right\},
\end{aligned}
$$

which turns out to be independent of $x_{6}, x_{8}, x_{10}$, due to the external momenta being on-shell, and 


$$
\begin{aligned}
\mathcal{A}_{2}(a)= & -i h \pi \frac{16 \sqrt{3}}{M^{3 / 2}} \alpha\left|\Psi_{\bar{q} q_{1}}(0)\right| \epsilon_{\mu}\left(k_{1}\right) \epsilon_{\nu}\left(k_{2}\right) \epsilon_{\rho}(P)\left[e_{q} \sin 2 \theta_{W} K_{\gamma \gamma \gamma}+\left(\frac{M}{M_{Z}}\right)^{2} c_{V}^{q} K_{Z \gamma \gamma}\right] \Theta_{3}^{\mu \nu \rho}\left[a ; P,-k_{1},-k_{2}\right] \\
= & -i h \pi 8 \sqrt{3 M} \alpha\left|\Psi_{\bar{q} q_{1}}(0)\right| \epsilon_{\mu}\left(k_{1}\right) \epsilon_{\nu}\left(k_{2}\right) \epsilon_{\rho}(P)\left[e_{q} \sin 2 \theta_{W} K_{\gamma \gamma \gamma}+\left(\frac{M}{M_{Z}}\right)^{2} c_{V}^{q} K_{Z \gamma \gamma}\right] \\
& \times\left\{\left(k_{2}-k_{1}\right)^{\rho}\left(\theta^{\mu \nu}-2 g^{\mu \nu} \frac{\left(k_{1} \theta k_{2}\right)}{M^{2}}\right)\right. \\
& +2 g^{\mu \rho}\left(\left(k_{1} \theta\right)^{\nu}-2 k_{1}^{\nu} \frac{\left(k_{1} \theta k_{2}\right)}{M^{2}}\right)+2 g^{\nu \rho}\left(\left(k_{2} \theta\right)^{\mu}+2 k_{2}^{\mu} \frac{\left(k_{1} \theta k_{2}\right)}{M^{2}}\right)-(a-1)\left[(P \theta)^{\rho}\left(g^{\mu \nu}-\frac{2}{M^{2}} k_{2}^{\mu} k_{1}^{\nu}\right)\right. \\
& \left.\left.+\left(k_{1} \theta\right)^{\mu}\left(g^{\nu \rho}+\frac{k_{1}^{\rho} k_{1}^{\nu}-k_{2}^{\rho} k_{1}^{\nu}}{M^{2}}\right)+\left(k_{2} \theta\right)^{\nu}\left(g^{\mu \rho}+\frac{k_{2}^{\rho} k_{2}^{\mu}-k_{1}^{\rho} k_{2}^{\mu}}{M^{2}}\right)\right]\right\} .
\end{aligned}
$$

The coupling constants appearing in the above amplitudes are evaluated at the $M_{Z}$ scale $[4,20]$. Here $P=k_{1}+k_{2}$, $\left(k_{i} \theta\right)^{\mu}=k_{i \nu} \theta^{\nu \mu}$ and $k_{1} \theta k_{2}=k_{1 \mu} \theta^{\mu \nu} k_{2 \nu}$, while $M$ and $P$ are the mass and the total momentum of the discussed quarkonium state, respectively.

Both of the above amplitudes satisfy separately the usual Ward identities that follow from gauge invariance and the pole structure of the diagrams. Note that this would have been a problematic issue had we included in our deformed fermion lagrangian terms that contributed to the tree level fermion propagator. It is also worth noticing that, despite the dependence of the Feynman rules of eqs. (24) and (25) on the parameters $x_{6}, x_{8}, x_{10}$-where $x_{8}$ and $x_{10}$ were associated to $\mathrm{C}, \mathrm{T}$ violating contributions in the strong and hypercharge sectors -this dependence disappears in the amplitude $\mathcal{A}_{1}$ after evaluating the external momenta on-shell. In fact, the amputated Green function evaluated at generic momenta can be seen to depend on $x_{6}, x_{8}, x_{10}$, thus not contradicting the expectations of ref. [19], which stated the necessity of adding a term involving $\gamma^{\mu \nu \rho}$ to get one-loop renormalisability. Indeed, despite not contributing to the calculated amplitude, the associated terms in the basis of eq. (12) might influence other S-matrix elements or may be relevant to renormalisability properties. It is also worth to notice the dependence of the amplitude $\mathcal{A}_{1}$ in eq. (31) on the parameter $x_{11}$, which induces CPT violations in the weak sector. However, as can be seen from the diagrams of figure 2 , the noncommutative vertices with fermion fields appearing in them only involve photons - no weak fields-, so that our computation is not affect by CPT violating interactions.

In ref. [12], when computing the amplitudes for the above diagrams in the nmNCSM without the $a$-deformation of eq. (1) and without the additional deformations, $x_{i}$, of the fermionic terms, (22), it was found that the on-shell, amputated $\mathcal{A}_{1}$ amplitude, corresponding to the sum of the five quark-exchange diagrams in Fig. 2, was proportional to the on-shell, amputated amplitude $\mathcal{A}_{2}$ of the boson-exchange Green functions represented in fig. 3, which could hint at a possible symmetry. This was made manifest by the fact that the S-matrix amplitudes in ref. [12] are given by some factor squared. In the present calculation, for $a=3$, which is the one making the gauge sector of deformed nmNCSM one-loop renormalisable, this result cannot be recovered for any value of the free deformation parameters $x_{4}, x_{5}, x_{6}, x_{8}, x_{10}, x_{11}$; in fact proportionality is only achieved for $a=1$ and $x_{i}=0$. The fact that we eliminated some of the freedom parameters $x_{i}$ should not be relevant to this result, since we are concerned with on-shell, amputated amplitudes which are not affected by the field redefinitions that allowed us to eliminate some of the $x_{i}$.

With respect to the possibility of considering terms deforming the tree-level fermion propagators, which have been ignored here, we would like to point out again that they would not only modify the on-shell conditions, but also cast serious doubts about the validity of the usual procedure of constructing the S-matrix amplitudes in terms of amputated diagrams; further considerations on these issues are out of the scope of this paper.

\section{Quarkonia decay rate}

To obtain the quarkonia decay rates we have to compute

$$
\Gamma\left(\bar{q} q_{1} \rightarrow \gamma \gamma\right)=\frac{1}{2 E_{\bar{q} q_{1}}} \frac{1}{4 \pi^{2}} \int \frac{d^{3} k_{1}}{2 E_{1}} \frac{d^{3} k_{2}}{2 E_{2}} \delta^{4}\left(P-k_{1}-k_{2}\right) \frac{1}{2 s_{i}+1} \sum_{\text {spins }}|\mathcal{M}|^{2} \frac{1}{2},
$$

where $\mathcal{M}$ is the total amplitude, i.e. $\mathcal{M}=\mathcal{A}_{1}+\mathcal{A}_{2}$. Summing over the initial spins and averaging over their final values in the square absolute value of the amplitude gives 


$$
\begin{aligned}
& \sum_{\text {spins }}|\mathcal{M}|^{2}=\frac{3 e^{4} M^{3}|\Psi|^{2}}{\left(M^{2}-M_{Z}^{2}\right)^{2}}\left[A\left(\theta_{\alpha \beta} \theta^{\beta \alpha}\right)+B\left(\theta_{\alpha \rho} \theta^{\rho}{ }_{\beta} k_{1}^{\alpha} k_{2}^{\beta}\right)+C\left(\theta_{\alpha \rho} \theta^{\rho}{ }_{\beta} k_{1}^{\alpha} k_{1}^{\beta}+\theta_{\alpha \rho} \theta^{\rho}{ }_{\beta} k_{2}^{\alpha} k_{2}^{\beta}\right)+D\left(\theta_{\alpha \beta} k_{1}^{\alpha} k_{2}^{\beta}\right)^{2}\right] \\
& A=-\left[-2 C_{v} K_{\gamma \gamma Z} M^{2}+e_{q}^{2}\left(4 x_{4}+4 x_{5}-1\right)\left(M^{2}-M_{Z}^{2}\right)+2 e_{q} \sin \left(2 \theta_{W}\right) K_{\gamma \gamma \gamma}\left(M^{2}-M_{Z}^{2}\right)\right]^{2}, \\
& B=\frac{8}{M^{2}}\left[\left(5 a^{2}-22 a+25\right) C_{v}^{2} K_{\gamma \gamma Z}^{2} M^{4}\right. \\
& +2 e_{q}^{2} C_{v} K_{\gamma \gamma Z}\left(M^{2}-M_{Z}^{2}\right) M^{2}\left(-28 x_{4}-28 x_{5}+a\left(12 x_{4}+12 x_{5}-4 x_{11}-3\right)+12 x_{11}+7\right) \\
& +e_{q}^{2} \sin ^{2}\left(2 \theta_{W}\right) K_{\gamma \gamma \gamma}^{2}\left(M^{2}-M_{Z}^{2}\right)^{2}\left(5 a^{2}-22 a+25\right) \\
& +2 e_{q}^{4}\left(16 x_{4}^{2}+8\left(4 x_{5}-2 x_{11}-1\right) x_{4}+18 x_{5}^{2}+2 x_{11}^{2}+4 x_{11}-4 x_{5}\left(3 x_{11}+2\right)+1\right)\left(M^{2}-M_{Z}^{2}\right)^{2} \\
& -2 \sin \left(2 \theta_{W}\right) K_{\gamma \gamma \gamma}\left(M^{2}-M_{Z}^{2}\right)\left(e_{q}\left(5 a^{2}-22 a+25\right) C_{v} K_{\gamma \gamma Z} M^{2}\right. \\
& \left.\left.+e_{q}^{3}\left(-28 x_{4}-28 x_{5}+a\left(12 x_{4}+12 x_{5}-4 x_{11}-3\right)+12 x_{11}+7\right)\left(M^{2}-M_{Z}^{2}\right)\right)\right] \text {, } \\
& C=\frac{8}{M^{2}}\left[\left(3 a^{2}-10 a+11\right) C_{v}^{2} K_{\gamma \gamma Z}^{2} M^{4}\right. \\
& +2 e_{q}^{2} C_{v} K_{\gamma \gamma Z}\left(M^{2}-M_{Z}^{2}\right) M^{2}\left(-12 x_{4}-11 x_{5}+a\left(4 x_{4}+5 x_{5}-3 x_{11}-1\right)+5 x_{11}+3\right) \\
& +e_{q}^{2} \sin ^{2}\left(2 \theta_{W}\right) K_{\gamma \gamma \gamma}^{2}\left(M^{2}-M_{Z}^{2}\right)^{2}\left(3 a^{2}-10 a+11\right) \\
& +e_{q}^{4}\left(M^{2}-M_{Z}^{2}\right)^{2}\left(16 x_{4}^{2}+8\left(3 x_{5}-x_{11}-1\right) x_{4}+12 x_{5}^{2}+4 x_{11}^{2}+2 x_{11}-2 x_{5}\left(4 x_{11}+3\right)+1\right) \\
& -2 \sin \left(2 \theta_{W}\right) K_{\gamma \gamma \gamma}\left(M^{2}-M_{Z}^{2}\right)\left(e_{q}\left(3 a^{2}-10 a+11\right) C_{v} K_{\gamma \gamma Z} M^{2}\right. \\
& \left.\left.+e_{q}^{3}\left(-12 x_{4}-11 x_{5}+a\left(4 x_{4}+5 x_{5}-3 x_{11}-1\right)+5 x_{11}+3\right)\left(M^{2}-M_{Z}^{2}\right)\right)\right], \\
& D=-\frac{16}{M^{4}}\left[\left(3 a^{2}-14 a+15\right) C_{v}^{2} K_{\gamma \gamma Z}^{2} M^{4}\right. \\
& +4 e_{q}^{2} C_{v} K_{\gamma \gamma Z}\left(M^{2}-M_{Z}^{2}\right) M^{2}\left(-8 x_{4}-8 x_{5}+a\left(4 x_{4}+4 x_{5}-2 x_{11}-1\right)+6 x_{11}+2\right) \\
& +e_{q}^{2} \sin ^{2}\left(2 \theta_{W}\right) K_{\gamma \gamma \gamma}^{2}\left(M^{2}-M_{Z}^{2}\right)^{2}\left(3 a^{2}-14 a+15\right) \\
& +e_{q}^{4}\left(M^{2}-M_{Z}^{2}\right)^{2}\left(16 x_{4}^{2}+8\left(4 x_{5}-4 x_{11}-1\right) x_{4}+20 x_{5}^{2}+4 x_{11}^{2}+8 x_{11}-8 x_{5}\left(3 x_{11}+1\right)+1\right) \\
& -2 \sin \left(2 \theta_{W}\right) K_{\gamma \gamma \gamma}\left(M^{2}-M_{Z}^{2}\right)\left(e_{q}\left(3 a^{2}-14 a+15\right) C_{v} K_{\gamma \gamma Z} M^{2}\right. \\
& \left.\left.+2 e_{q}^{3}\left(-8 x_{4}-8 x_{5}+a\left(4 x_{4}+4 x_{5}-2 x_{11}-1\right)+6 x_{11}+2\right)\left(M^{2}-M_{Z}^{2}\right)\right)\right] .
\end{aligned}
$$

Considering the quarkonia at rest and using the phase space integrals

$$
\begin{aligned}
\left(-\theta^{2}\right) \int \frac{d^{3} k_{1}}{2 E_{1}} \frac{d^{3} k_{2}}{2 E_{2}} \delta^{4}\left(P-k_{1}-k_{2}\right) & =\frac{2}{\Lambda_{\mathrm{NC}}^{4}}\left(\vec{B}_{\theta}^{2}-\vec{E}_{\theta}^{2}\right) \frac{\pi}{2}, \\
\left(P \theta^{2} P\right) \int \frac{d^{3} k_{1}}{2 E_{1}} \frac{d^{3} k_{2}}{2 E_{2}} \delta^{4}\left(P-k_{1}-k_{2}\right) & =\frac{M^{2}}{\Lambda_{\mathrm{NC}}^{4}}\left(\vec{E}_{\theta}^{2}\right) \frac{\pi}{2}, \\
\int \frac{d^{3} k_{1}}{2 E_{1}} \frac{d^{3} k_{2}}{2 E_{2}} \delta^{4}\left(P-k_{1}-k_{2}\right)\left(k_{1} \theta^{2} k_{1}\right) & =\frac{M^{2}}{\Lambda_{\mathrm{NC}}^{4}}\left(\vec{E}_{\theta}^{2}+\vec{B}_{\theta}^{2}\right) \frac{\pi}{12}, \\
\int \frac{d^{3} k_{1}}{2 E_{1}} \frac{d^{3} k_{2}}{2 E_{2}} \delta^{4}\left(P-k_{1}-k_{2}\right)\left(k_{1} \theta k_{2}\right)^{2} & =\frac{M^{4}}{\Lambda_{\mathrm{NC}}^{4}}\left(\vec{E}_{\theta}^{2}\right) \frac{\pi}{24},
\end{aligned}
$$

starting from (33), for general $a, x_{i}$, we obtain the following decay rate

$$
\Gamma^{\mathrm{nmNCSM}}\left(\bar{q} q_{1} \rightarrow \gamma \gamma\right)=\frac{\pi}{24} \frac{\alpha^{2} M^{2}|\Psi|^{2}}{\left(M^{2}-M_{Z}^{2}\right)^{2} \Lambda_{\mathrm{NC}}^{4}}\left[\left(24 A+4 M^{2}(B+C)+M^{4} D\right) \vec{E}_{\theta}^{2}-\left(24 A+2 M^{2}(B-2 C)\right) \vec{B}_{\theta}^{2}\right] .
$$


The above rate turns into eq.(12) from [12] for $a=1, x_{i}=0$. We would like to analyse eq. (40) and study the effects of having added to the action of the nmNCSM the extra terms that have been discussed in the previous sections, in order to compare the results with those calculated in ref. [12]. In order to obtain some numerical values, we will look for the maxima and minima of the decay rates in the allowed region for $K_{Z \gamma \gamma}$ and $K_{\gamma \gamma \gamma}$-see figure 1- with the assumption that the $x_{i}$ parameters are "natural" and only take values between zero and one, and with two possible scenarios for the dimensionless constants $\vec{E}_{\theta}^{2}$ and $\vec{B}_{\theta}^{2}$ : either both of them are of order one (space-time and space-space noncommutativity), or $\vec{E}_{\theta}^{2}=0$ and $\vec{B}_{\theta}^{2}$ is of order one (only space-space noncommutativity). Moreover, as was done in ref. [12], we will consider that the scale of noncommutativity varies between $\Lambda_{\mathrm{NC}}=0.25 \mathrm{TeV}$ and $\Lambda_{\mathrm{NC}}=1 \mathrm{TeV}$.

In order to compute branching ratios, we use the following data taken from [20]: in the $J / \psi$ case, $\Gamma^{\exp .}(J / \psi \rightarrow$ $\left.e^{+} e^{-}\right)=(5.55 \pm 0.14 \pm 0.02) \mathrm{keV}$ and $\Gamma_{\text {tot }}^{\text {exp. }}(\Upsilon)=(93.2 \pm 2.1) \mathrm{keV}$, whereas for the $\Upsilon$ case, $\Gamma^{\exp .}\left(\Upsilon \rightarrow e^{+} e^{-}\right)=$ $(1.340 \pm 0.018) \mathrm{keV}$ and $\Gamma_{\text {tot }}^{\text {exp. }}(\Upsilon)=(54.02 \pm 1.25) \mathrm{keV}$. Recall that the wavefunction at the origin $\Psi(0)$ is related to the lepton decay rate by eq. (30).

First, since in the minimal NCSM (mNCSM) there are no $Z \gamma \gamma$ and $\gamma \gamma \gamma$ couplings, the formula (40) can be used to recover the branching rations in the minimal NCSM -see ref. [12], eqs. (19,20)- by taking $K_{Z \gamma \gamma \gamma}=K_{\gamma \gamma \gamma}=0$, which yields, for $\vec{E}_{\theta}^{2}=\vec{B}_{\theta}^{2}=1$ and $0.25 \mathrm{TeV} \leq \Lambda_{\mathrm{NC}} \leq 1 \mathrm{TeV}$,

$$
5.1 \cdot 10^{-13} \lesssim B R_{[J / \psi \rightarrow \gamma \gamma]}^{m N C S M} \lesssim 1.3 \cdot 10^{-10}, \quad 4.6 \cdot 10^{-12} \lesssim B R_{[\Upsilon \rightarrow \gamma \gamma]}^{m N C S M} \lesssim 1.2 \cdot 10^{-9} .
$$

The values at $\vec{E}_{\theta}^{2}=0, \vec{B}_{\theta}^{2}=1$ are suppressed by a factor of $3 / 10$.

In ref. [12], the computation in undeformed nmNCSM was also done for $\vec{E}_{\theta}^{2}=\vec{B}_{\theta}^{2}=1$, yielding

$$
\frac{\Gamma^{a=1}\left(\bar{q} q_{1} \rightarrow \gamma \gamma\right)}{\Gamma\left(\bar{q} q_{1} \rightarrow \ell^{+} \ell^{-}\right)}=\frac{5}{24} e_{q}^{2}\left(\frac{M}{\Lambda_{\mathrm{NC}}}\right)^{4}\left[1-\frac{2}{e_{q}} \sin 2 \theta_{W} K_{\gamma \gamma \gamma}-\frac{2}{e_{q}^{2}}\left(\frac{M}{M_{Z}}\right)^{2} c_{V}^{q} K_{Z \gamma \gamma}\right]^{2} .
$$

This corresponds to setting $a=1, x_{i}=0$ in the deformed version of the nmNCSM of this paper.

Computing the maximal values of the above rate for $0.25 \mathrm{TeV} \leq \Lambda_{\mathrm{NC}} \leq 1 \mathrm{TeV}$ yields

$$
3.1 \cdot 10^{-12} \lesssim B R_{[J / \psi \rightarrow \gamma \gamma], \text { max }}^{a=1, x_{i}=0} \lesssim 7.8 \cdot 10^{-10}, \quad 1.7 \cdot 10^{-11} \lesssim B R_{[\Upsilon \rightarrow \gamma \gamma], \text { max }}^{a=1, x_{i}=0} \lesssim 4.3 \cdot 10^{-9},
$$

as was obtained in eqs. $(23,24)$ from [12].

On the other side, the minimal values in the same range of $\Lambda_{\mathrm{NC}}$ are

$$
2.3 \cdot 10^{-13} \lesssim B R_{[J / \psi \rightarrow \gamma \gamma], \text { min }}^{a=1, x_{i}=0} \lesssim 5.9 \cdot 10^{-11}, \quad 1.0 \cdot 10^{-26} \lesssim B R_{[\Upsilon \rightarrow \gamma \gamma], \text { min }}^{a=1, x_{i}=0} \lesssim 2.6 \cdot 10^{-24} .
$$

By taking $\vec{E}_{\theta}^{2}=0, \vec{B}_{\theta}^{2}=1$ all the results are suppressed by a factor of $3 / 10$.

To see the effect of adding to the action the extra pure gauge term associated with the choice $a=3$ in eq. (5), corresponding to the deformed nmNCSM with renormalisable pure gauge interactions, we can start from (40) and fix $a=3$ and $x_{4}=x_{5}=x_{11}=0$. The resulting expression is more complicated than (42),

$$
\begin{aligned}
\frac{\Gamma^{a=3}\left(\bar{q} q_{1} \rightarrow \gamma \gamma\right)}{\Gamma\left(\bar{q} q_{1} \rightarrow \ell^{+} \ell^{-}\right)}=\frac{M^{4}}{48 e_{q}^{2} \Lambda_{\mathrm{NC}}^{4}\left(M^{2}-M_{Z}^{2}\right)^{2}}\left(R \vec{B}_{\theta}^{2}+S \vec{E}_{\theta}^{2}\right), \\
R=3\left(M^{2}-M_{Z}^{2}\right)^{2} e_{q}^{4}+36 \sin ^{2}\left(2 \theta_{W}\right) K_{\gamma \gamma \gamma}^{2}\left(M^{2}-M_{Z}^{2}\right)^{2} e_{q}^{2}+20 c_{V}^{q} M^{2} K_{Z \gamma \gamma}\left(M^{2}-M_{Z}^{2}\right) e_{q}^{2} \\
\quad-4 \sin \left(2 \theta_{W}\right) K_{\gamma \gamma \gamma}\left(M^{2}-M_{Z}^{2}\right)\left(5\left(M^{2}-M_{Z}^{2}\right) e_{q}{ }^{2}+18 c_{V}^{q} M^{2} K_{Z \gamma \gamma}\right) e_{q}+36 c_{V}^{q} M^{4} K_{Z \gamma \gamma}^{2} \\
S=7\left(M^{2}-M_{Z}^{2}\right)^{2} e_{q}^{4}+36 \sin ^{2}\left(2 \theta_{W}\right) K_{\gamma \gamma \gamma}^{2}\left(M^{2}-M_{Z}^{2}\right)^{2} e_{q}^{2}-20 c_{V}^{q} M^{2} K_{Z \gamma \gamma}\left(M^{2}-M_{Z}^{2}\right) e_{q}^{2} \\
\quad+4 \sin \left(2 \theta_{W}\right) K_{\gamma \gamma \gamma}\left(M^{2}-M_{Z}^{2}\right)\left(5 e_{q}^{2}\left(M^{2}-M_{Z}^{2}\right)-18 c_{V}^{q} M^{2} K_{Z \gamma \gamma}\right) e_{q}+36 c_{V}^{q}{ }^{2} M^{4} K_{Z \gamma \gamma}^{2} .
\end{aligned}
$$

The maximal values in the allowed regions for $K_{Z \gamma \gamma}$ and $K_{\gamma \gamma \gamma}$ of the branching ratios, for $0.25 \mathrm{TeV} \leq \Lambda_{\mathrm{NC}} \leq 1 \mathrm{TeV}$, $\vec{E}_{\theta}^{2}=\vec{B}_{\theta}^{2}=1$ become

$$
2.4 \cdot 10^{-12} \lesssim B R_{[J / \psi \rightarrow \gamma \gamma], \max }^{a=3, x_{i}=0} \lesssim 6.3 \cdot 10^{-10}, \quad 7.5 \cdot 10^{-11} \lesssim B R_{[\Upsilon \rightarrow \gamma \gamma], \text { max }}^{a=3, x_{i}=0} \lesssim 1.9 \cdot 10^{-8},
$$

whereas for the minimal values we get

$$
5.1 \cdot 10^{-13} \lesssim B R_{[J / \psi \rightarrow \gamma \gamma], \min }^{a=3, x_{i}=0} \lesssim 1.3 \cdot 10^{-10}, \quad 4.6 \cdot 10^{-12} \lesssim B R_{[\Upsilon \rightarrow \gamma \gamma], \min }^{a=3, x_{i}=0} \lesssim 1.2 \cdot 10^{-9}
$$


They remain more or less in the same order of magnitude as those of eq. (43), safe for the minimal values of the $\Upsilon$ branching ratio, which are hugely increased. In particular, the minimal values are always above those of the mNCSM of eq. (41). In the case $\vec{E}_{\theta}^{2}=0, \vec{B}_{\theta}^{2}=1$ the rates are again suppressed but in a different way:

$$
\begin{gathered}
1.9 \cdot 10^{-12} \lesssim B R_{[J / \psi \rightarrow \gamma \gamma], \text { max }}^{a=3, x_{i}=0} \lesssim 4.8 \cdot 10^{-10}, \quad 2.3 \cdot 10^{-11} \lesssim B R_{[\Upsilon \rightarrow \gamma \gamma], \text { max }}^{a=3, x_{i}=0} \lesssim 5.9 \cdot 10^{-9}, \\
3.5 \cdot 10^{-14} \lesssim B R_{[J / \psi \rightarrow \gamma \gamma], \text { min }}^{a=3, x_{i}=0} \lesssim 9.0 \cdot 10^{-12}, \quad 1.0 \cdot 10^{-13} \lesssim B R_{[\Upsilon \rightarrow \gamma \gamma], \text { min }}^{a=3, x_{i}=0} \lesssim 2.6 \cdot 10^{-11} .
\end{gathered}
$$

In particular, the minimal values are no longer above the ones corresponding to the mNCSM with $\vec{E}_{\theta}^{2}=0, \vec{B}_{\theta}^{2}=1$, but there is still a dramatic increase in the minimal possible values for the $\Upsilon$ branching ratio.

So far, the effect of the extra pure gauge term in the deformed nmNCSM with respect to the undeformed version is essentially an enhancement of the minimal allowed values of the $\Upsilon$ branching ratio.

Next we move on to see the effect of the extra terms in the fermionic action depending on $x_{4}, x_{5}, x_{11}$, when these parameters take natural values between -1 and 1 . We set to calculate the maximal and minimal values that the branching ratios obtained from eq. (40) can have for $x_{i} \in\{-1,1\}$ within the allowed values of $K_{Z \gamma \gamma}$ and $K_{\gamma \gamma \gamma}$.

In the case $\vec{E}_{\theta}^{2}=\vec{B}_{\theta}^{2}=1$, we obtain the following results, as before for scales $0.25 \mathrm{TeV} \leq \Lambda_{\mathrm{NC}} \leq 1 \mathrm{TeV}$ :

$$
\begin{aligned}
& 4.5 \cdot 10^{-11} \lesssim B R_{[J / \psi \rightarrow \gamma \gamma], \text { max }}^{a=3,\left|x_{i}\right| \sim 1} \lesssim 1.2 \cdot 10^{-8}, \quad 5.6 \cdot 10^{-10} \lesssim B R_{[\Upsilon \rightarrow \gamma \gamma], \text { max }}^{a=3,\left|x_{i}\right| \sim 1} \lesssim 1.4 \cdot 10^{-7}, \\
& B R_{[J / \psi \rightarrow \gamma \gamma], \text { min }}^{a=3,\left|x_{i}\right| \sim 1} \sim 0, \quad B R_{[\Upsilon \rightarrow \gamma \gamma], \text { min }}^{a=3,\left|x_{i}\right| \sim 1} \sim 0 .
\end{aligned}
$$

As an explanation of the previous values, for example the first expression $4.5 \cdot 10^{-11} \lesssim B R_{[J / \psi \rightarrow \gamma \gamma], \text { max }}^{a=3,\left|x_{i}\right| \sim 1} \lesssim 1.2 \cdot 10^{-8}$ means that the maximum of the $J / \psi$ branching ratio, for the range of scales $0.25 \mathrm{TeV} \leq \Lambda_{\mathrm{NC}} \leq 1 \mathrm{TeV}$, for all $x_{i} \in\{-1,1\}$ and for all allowed $K_{Z \gamma \gamma}$ and $K_{\gamma \gamma \gamma}$, varies between $4.5 \cdot 10^{-11}$-reached at the scale $\Lambda_{\mathrm{NC}}=1 \mathrm{TeV}$, and $1.2 \cdot 10^{-8}$, reached at $\Lambda_{\mathrm{NC}}=0.25 \mathrm{TeV}$. Note that the dependency of the rates on $\Lambda_{\mathrm{NC}}$ factorises, as seen in eq. (40). Each value is reached for a particular value of $x_{i}, K_{Z \gamma \gamma}, K_{\gamma \gamma \gamma}$ :

$$
\begin{aligned}
& \text { Maxima } \\
& \qquad \begin{array}{l}
J / \psi: x_{4}=-1.00, x_{5}=-1.00, x_{11}=1.00, K_{Z \gamma \gamma}=-0.254, K_{\gamma \gamma \gamma}=0.129, \\
\Upsilon: x_{4}=-1.00, x_{5}=-1.00, x_{11}=1.00, K_{Z \gamma \gamma}=0.00950, K_{\gamma \gamma \gamma}=-0.576,
\end{array}
\end{aligned}
$$

whereas for the minima the numerical results are not reliable due to precision issues. Figures 4 through 7 show the resulting branching ratios as functions of $K_{Z \gamma \gamma}$ and $K_{\gamma \gamma \gamma}$ for the particular values of the $x_{i}$ that yielded the above maxima and (approximate) minima, respectively, at the scale $\Lambda_{\mathrm{NC}}=1 \mathrm{TeV}$. The maxima of figures 4 and 5 correspond to the values appearing at the left of the inequalities in eq. (49). The minima of figures 6 and 7 are practically zero.

For completeness we show the corresponding results in the $\vec{E}_{\theta}^{2}=0, \vec{B}_{\theta}^{2}=1$ case:

$$
\begin{aligned}
& 2.0 \cdot 10^{-11} \lesssim B R_{[J / \psi \rightarrow \gamma \gamma], \max }^{a=3,\left|x_{i}\right| \sim 1} \lesssim 5.1 \cdot 10^{-9}, \quad 2.0 \cdot 10^{-10} \lesssim B R_{[\Upsilon \rightarrow \gamma \gamma], \text { max }}^{a=3,\left|x_{i}\right| \sim 1} \lesssim 5.0 \cdot 10^{-8}, \\
& B R_{[J / \psi \rightarrow \gamma \gamma], \text { min }}^{a=3,\left|x_{i}\right| \sim 1} \sim 0, \quad B R_{[\Upsilon \rightarrow \gamma \gamma], \text { min }}^{a=3,\left|x_{i}\right| \sim 1} \sim 0,
\end{aligned}
$$

The previous values are reached for the following values of the parameters:

$$
\begin{aligned}
& \text { Maxima } \\
& \qquad / \psi: x_{4}=-1.00, x_{5}=-1.00, x_{11}=-1.00, K_{Z \gamma \gamma}=0.00950, K_{\gamma \gamma \gamma}=-0.576, \\
& \Upsilon: x_{4}=1.00, x_{5}=1.00, x_{11}=1.00, K_{Z \gamma \gamma}=0.00950, K_{\gamma \gamma \gamma}=-0.576,
\end{aligned}
$$

where again we do not display the position of the minima since our numerical results are not reliable. The effect of the $x_{i}$ terms is clearly to allow for much larger maximum values and much lower minimum values of the branching ratios with respect to the results in the undeformed nmNCSM or the mNCSM. The maximum values are increased up to 2 orders of magnitude with respect to the mNCSM result of eq. (41) and one order of magnitude with respect to the results in eq. (43) corresponding to the undeformed version of the nmNCSM.

Despite the fact that the allowed values for the minima experience an important decrease, for typical values of the parameters $K_{Z \gamma \gamma}, K_{\gamma \gamma \gamma}, x_{4}, x_{5}, x_{11}$ the rates are enhanced with respect to the mNCSM result. To justify this claim we have computed the branching ratios for random values of the above parameters in the allowed region for $K_{Z \gamma \gamma}, K_{\gamma \gamma \gamma}$ displayed in figure 1 and for $x_{i} \in\{-1,1\}$. For up to one million configurations of these parameters, at 


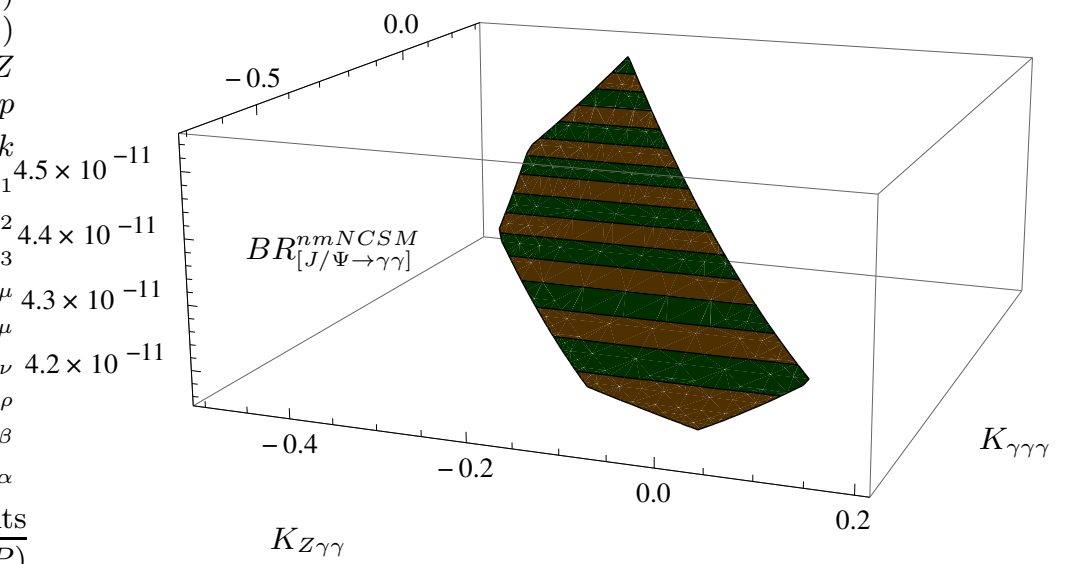

FIG. 4: $J / \Psi \rightarrow \gamma \gamma$ branching ratio as a function of $K_{Z \gamma \gamma}$ and $K_{\gamma \gamma \gamma}$, for $\vec{E}_{\theta}^{2}, \vec{B}_{\theta}^{2} \sim 1, x_{4}=x_{5}=-x_{11}=-1$, at the scale $\Lambda_{\mathrm{NC}}=1 \mathrm{TeV}$

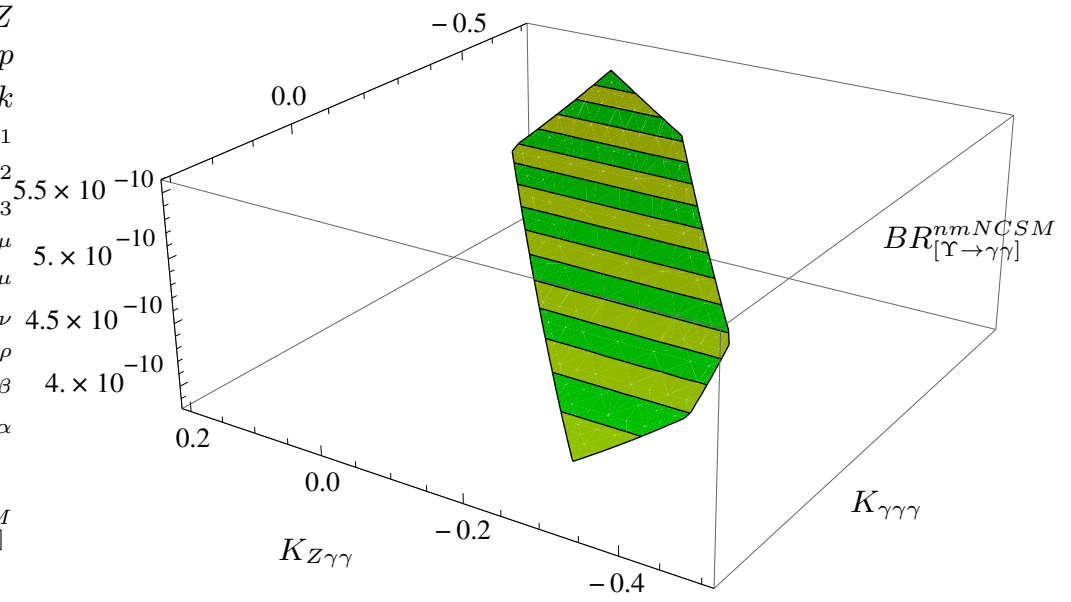

FIG. 5: $\Upsilon \rightarrow \gamma \gamma$ branching ratio as a function of $K_{Z \gamma \gamma}$ and $K_{\gamma \gamma \gamma}$, for $\vec{E}_{\theta}^{2}, \vec{B}_{\theta}^{2} \sim 1, x_{4}=x_{5}=-x_{11}=-1$, at the scale $\Lambda_{\mathrm{NC}}=1 \mathrm{TeV}$

the scale $\Lambda_{\mathrm{NC}}=1 \mathrm{TeV}$, we found that, for $\vec{E}_{\theta}^{2}=\vec{B}_{\theta}^{2}=1,\left(\vec{E}_{\theta}^{2}=0, \vec{B}_{\theta}^{2}=1\right), 96(88) \%$ of the configurations yield $J / \psi$ branching ratios larger than their corresponding values in the mNCSM, whereas in the $\Upsilon$ case the percentages are $97(89) \%$. The percentage of configurations yielding a 10× increase over the mNCSM values are $44(42) \%$ in the $J / \psi$ case and $55(40) \%$ in the $\Upsilon$ case. There is a $50 \times$ increase for $2(4) \%$ of the $J / \psi$ configurations and for $4(3) \%$ of the $\Upsilon$ configurations.

We have also estimated the portion of the parameter space which, at the scale $\Lambda_{\mathrm{NC}}=1 \mathrm{TeV}$, yields values of the branching ratios which are larger than the maximum values in the undeformed version of the nmNCSM, which are given in eq. (43). The percentage of configurations that satisfy this requirement for $\vec{E}_{\theta}^{2}=\vec{B}_{\theta}^{2}=1,\left(\vec{E}_{\theta}^{2}=0, \vec{B}_{\theta}^{2}=1\right)$, is given in the $J / \psi$ case by $62(55) \%$, whereas in the $\Upsilon$ case it is equal to $84(67) \%$.

To complete our numerical estimates, we should consider the case when the parameters $x_{i}$ take values that are necessarily non-natural, i.e, not necessarily restricted to be between -1 and 1 . An analysis of the dependence of the branching ratios on the parameters $K_{Z \gamma \gamma}, K_{\gamma \gamma \gamma}, x_{i}$ shows that, independently of the $x_{i}$, the branching ratios as a function of $K_{Z \gamma \gamma}$ and $K_{\gamma \gamma \gamma}$ define a concave parabolic surface (in the $J / \Psi$ case) or a hyperbolic surface ( $\Upsilon$ case). Thus the maxima always appear in the boundary of the region of the parameter space, as has happened with our results in eqs. (50) and (52). It is clear that allowing a wider range of the $x_{i}$ will directly yield greater maxima; since 


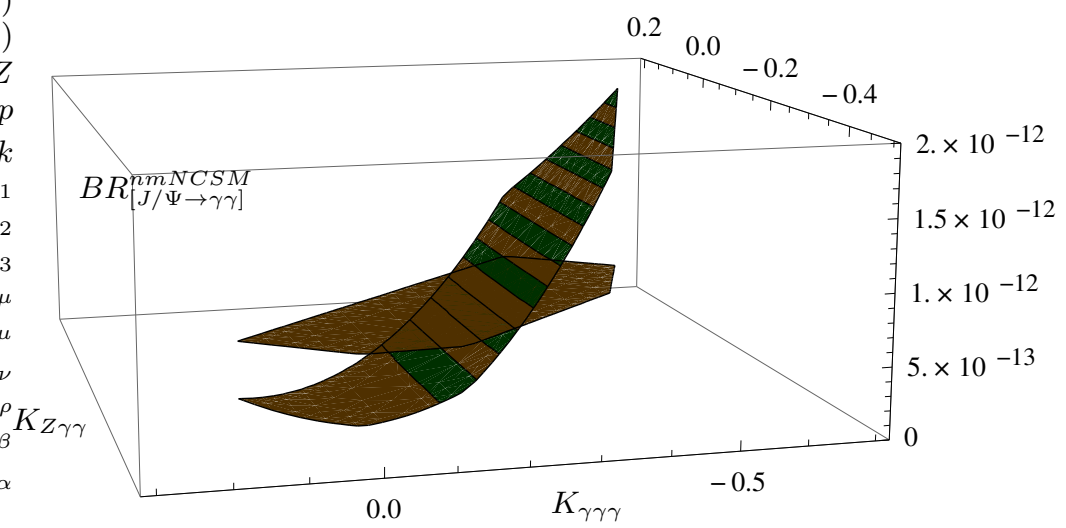

FIG. 6: $J / \Psi \rightarrow \gamma \gamma$ branching ratio as a function of $K_{Z \gamma \gamma}$ and $K_{\gamma \gamma \gamma}$, for $\vec{E}_{\theta}^{2}, \vec{B}_{\theta}^{2} \sim 1, x_{4}=0.25, x_{5}=-2.85 \cdot 10^{-7}, x_{11}=$ $2.86 \cdot 10^{-7}$, at the scale $\Lambda_{\mathrm{NC}}=1 \mathrm{TeV}$. The horizontal plane represents the mNCSM branching ratio of $5.1 \cdot 10^{-13}$.

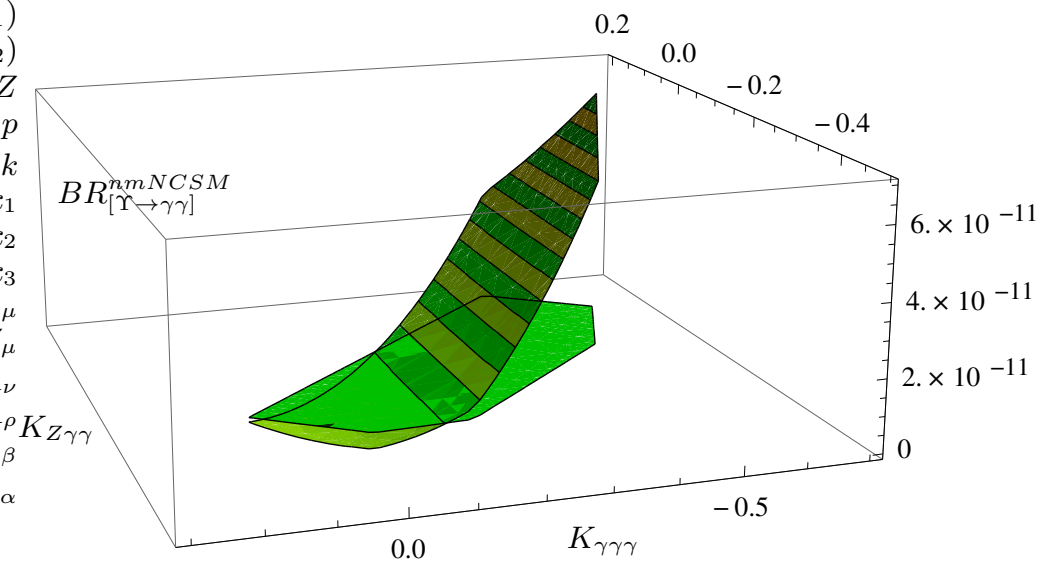

FIG. 7: $\Upsilon \rightarrow \gamma \gamma$ branching ratio as a function of $K_{Z \gamma \gamma}$ and $K_{\gamma \gamma \gamma}$, for $\vec{E}_{\theta}^{2}, \vec{B}_{\theta}^{2} \sim 1, x_{4}=0.25, x_{5}=-2.85 \cdot 10^{-7}, x_{11}=2.86 \cdot 10^{-7}$, at the scale $\Lambda_{\mathrm{NC}}=1 \mathrm{TeV}$. The horizontal plane represents the mNCSM branching ratio of $4.6 \cdot 10^{-12}$.

the dependence of the branching ratios is quadratic in the $x_{i}$, we expect that an increase of an order of magnitude in the range of the $x_{i}$ yields a two orders of magnitude increase in the maximum values of the branching ratios. By doing some numerical computations this seems to be roughly the case (modulo a factor between 0.45 and 0.77 ). In particular, taking $x_{i} \in\{-100,100\}$, we get, for $\vec{E}_{\theta}^{2}=\vec{B}_{\theta}^{2}=1$ and for scales $0.25 \mathrm{TeV} \leq \Lambda_{\mathrm{NC}} \leq 1 \mathrm{TeV}$ :

$$
3.5 \cdot 10^{-7} \lesssim B R_{[J / \psi \rightarrow \gamma \gamma], \max }^{a=3,\left|x_{i}\right| \sim 100} \lesssim 9.4 \cdot 10^{-5}, \quad 3.2 \cdot 10^{-6} \lesssim B R_{[\Upsilon \rightarrow \gamma \gamma], \max }^{a=3,\left|x_{i}\right| 100} \lesssim 8.1 \cdot 10^{-4} .
$$

The values at the scale $\Lambda=0.25 \mathrm{TeV}$ are within the experimental bounds: $B R(J / \psi \rightarrow \gamma \gamma)<2.2 \times 10^{-5}[20]$ and $B R(\Upsilon \rightarrow \gamma \gamma) \lesssim 10^{-4}[21]$

\section{DISCUSSION AND CONCLUSIONS}

In this paper we have introduced a deformed fermion lagrangian for the nmNCSM and we have applied it to the phenomenological estimate of the quarkonia decay rates into two photons. 
First of all, let us recall that the non-minimal version of the NCSM gauge sector includes traces over the representations of all the massive particle multiplets with different quantum numbers that appear in the total lagrangian of the model with covariant derivatives acting on them, i.e., with terms of the type $i \widehat{\psi} \star \hat{D} \star \widehat{\psi},\left(\hat{D}^{\mu} \widehat{\Phi}\right)^{\dagger} \star\left(\hat{D}_{\mu} \widehat{\Phi}\right),[4,5]$.

Second, we have argued why the use of the nmNCSM, and in particular its deformed version, should be favoured over the mNCSM; this is because of the proven renormalisability of the pure gauge sector and the existing hints that this property might not be spoilt when the effects of matter loops are taken into account. The motivations for introducing deformation terms in the fermion lagrangian come essentially from our lack of knowledge of the renormalisability properties of the matter sector of the full nmNCSM, and also from the fact that, for the gauge interactions, renormalisability -and even finiteness for the first noncommutative corrections- was only achieved by adding a deformation term to the starting lagrangian. Thus, our deformed fermion lagrangian should be taken as an effective lagrangian for the nmNCSM as long as the renormalisability properties are unknown. From the effective theory point of view, the values of the $x_{i}$ parameters could be constrained by experimental measurements.

It should be noted that the photon polarisation is known to be modified by noncommutativity, which causes vacuum birefringence [22], at least in the standard approach to noncommutative theories. This follows from computations of the one-loop photon self-energy. It should be interesting to analyse this issue in the enveloping algebra approach. Of course our new fermion-photon interactions, coming from the $x_{i}$-dependent terms, will also affect the photon polarisation at one-loop, which could be used for further experimental tests. However this is not straightforward since, according to ref. [23], the photon two point function, due to gauge invariance, will only be modified at order $\theta^{2}$ and beyond, so that a consistent computation would imply the use of SW maps up to $\mathrm{O}\left(\theta^{2}\right)$.

Aside from possible experimental measurements, there is hope that further investigations about renormalisability properties could impose constraints on the free deformation parameters $x_{i}$; it could happen that some or all of them were fixed uniquely, as happened for the gauge sector of the nmNCSM, and in that case the new fermion sector would not be effective but part of a theory well defined in the ultraviolet.

In deriving the deformed fermion lagrangian, we did not consider terms that altered the tree-level fermion propagator. This was done because, on the one hand, we should expect noncommutative effects to appear as weak quantum corrections, and thus we do not find desirable to break the usual Lorentz-invariance matter dispersion relation $p^{2}=m^{2}$ at tree level. On the other hand, we wanted to apply the framework to the computation of decay rates, which implies the calculation of S-matrix elements. In ordinary space-time one uses the LSZ formula, which relies on general properties of the pole structure of the Green functions of the theory (and in particular the 2-point function) which follow only from Poincaré invariance. In noncommutative space-time the usual Lorentz invariance does not hold, and thus a proper all-order definition of S-matrix elements seems challenging (in the case of noncommutative theories that do not make use of Seiberg-Witten maps, some advances have been done in this respect, see for example ref. [18]). Nevertheless, since we were computing S-matrix elements at tree level and we were not altering the tree-level 2-point function, we believe that the usual LSZ formalism should hold at this level. As a consistency check, our S-matrix amplitudes satisfy the usual Ward identities associated with U(1) gauge invariance, which are derived in ordinary space-time as a consequence of gauge symmetry and the pole structure of the diagrams. Nevertheless, a deeper understanding of the S-matrix and the LSZ formalism in noncommutative theories is still needed. We also recall that some of the terms considered in eq. (12) violate CPT exclusively in the weak sector; also, there appear C,T violations in the strong and hypercharge sectors. This could be of phenomenological interest for searches of Physics beyond the Standard Model; note that the violations are very small since they appear purely as noncommutative effects.

Concerning our results for the quarkonia decay amplitudes, it should be noticed first that the on-shell amplitudes turned out to be independent of the $\mathrm{C}$, $\mathrm{T}$ violating terms $t_{8}, t_{10}$; also, though they are dependent on $x_{11}$, which is associated to CPT violations in the weak sector, no CPT violating interactions contributed to the result. Ref. [19] argued that to get one-loop renormalisability in noncommutative QED, the term $t_{7}$ of eq. (12) should be added to the bare lagrangian. This does not conflict with the fact that the quarkonia decay amplitudes are not apparently influenced by the terms with three $\gamma^{\mu}$ matrices in eq. (12): first, we absorbed the contributions of $t_{7}$ in the terms $t_{4}-t_{6}, t_{8}, t_{10}, t_{11}$ by using field redefinitions, and, though the on-shell amplitudes did not depend on the parameters $x_{6}, x_{8}, x_{10}$, the amplitudes evaluated at arbitrary momenta did. With respet to the numerical results obtained for the decay rates, we showed that the effect of the extra gauge term in the action of the extended nmNCSM is essentially to raise the minimum allowed values of the $\Upsilon \rightarrow \gamma \gamma$ decay rate, masking the destructive contributions that were found in the undeformed nmNCSM in ref. [12]. However, when the new terms of our deformed fermion lagrangian are taken into account, this effect disappears, and both the minimum and maximum allowed values for the decay rates experience high decreases and increases, respectively.

Having very low allowed values for the quarkonia decay rates is no good news for the possibility of comparison with experiments in order to confirm or falsify the theoretical predictions: in principle, non-zero $q \bar{q}_{1} \rightarrow \gamma \gamma$ decay 
rates could be taken as a signal of noncommutativity, but if the models allow for extremely small values it would be difficult to discard them. However, the panorama is more promising because, despite the minimum possible values are very small, for most configurations of the parameters (between 88 and $97 \%$ at $\Lambda_{\mathrm{NC}}=1 \mathrm{TeV}$ for natural values of the parameters) we obtain branching ratios that are greater than the ones that were computed for the mNCSM. Furthermore, for a big portion of the parameter space (between 55 and $84 \%$ under the same conditions), the decay rates, computed from deformed nmNCSM, are actually larger than the maximum values that were found in the case of the undeformed nmNCSM.

Thus, in general we get constructive contributions to the decay rates and the model allows for greater values of the branching ratios that the ones that had been previously found; the maximum values increase by up to two orders of magnitude. Possible future studies of the renormalisability of the matter sector could help to restrict the allowed values in the parameter space, as happens in the gauge sector with the ambiguity parameter $a$ of eq. (5) forced to be equal to 3, and this could make the model more predictive or falsifiable. Again, we recall the result of ref. [19], which in the QED case argues that one-loop renormalisability demands to add only the term $t_{7}$ of eq. (12) to the lagrangian; this may also happen in the NCSM but the result cannot be directly extrapolated since, after the expansion with the SW map, the NCSM lagrangian is not given by a sum of lagrangians for the different gauge groups due to the appearance of interactions between the different gauge fields.

Todays existing experimental limit for the branching ratio of the $J / \psi \rightarrow \gamma \gamma$ decay can be found in "Review of particle physics," under the C symmetry violating modes, and is $B R(J / \psi \rightarrow \gamma \gamma)<2.2 \times 10^{-5}$ [20].

With respect to the $\Upsilon$ case, as it was commented in ref. [12], the existing limit for the branching ratio of the $\Upsilon \rightarrow \gamma \gamma$ decay comes from a very old CLEO-III experiment [21] and it indicates that with present data the detection of $B R(\Upsilon \rightarrow \gamma \gamma)$ below $10^{-4}$ would be hopeless.

As follows from eq. (53), the previous experimental bounds are reachable in our model at the scale $\Lambda=0.25 \mathrm{TeV}$ for values of $x_{i}$ tuned to be around 100, and for lower scales for higher values of the $x_{i}$. Thus there is some hope that the phenomenology of our model could be relevant.

Furthermore, despite the fact that all experiments including hadrons are extremely hard to perform and analyse due to the huge background signals, the large number of heavy quark-antiquark pairs harvested at LHCb; i.e. $10^{12}$ $B \bar{B}$ pairs per year [24], and probably $\left(10^{14} D \bar{D} ; 10^{18} K \bar{K}\right)$, give us hope that experimental branching ratios $B R(\Upsilon \rightarrow \gamma \gamma) \sim 10^{-9}$ and $B R(J / \psi \rightarrow \gamma \gamma) \sim 10^{-11}$ could be accessible, thus reaching our maximum predicted values for the rates for $\left|x_{i}\right| \sim 1$ (46-51). Certainly these experiments would produce at least reliable (and much lower) bounds; perhaps they could even measure these processes, depending on the scale of noncommutativity. We hope that the importance of a possible discovery of space-time non-commutativity will convince experimentalists to look for SM forbidden decays in hadronic physics.

\section{Acknowledgments}

The authors wish to thank Maja Buric, Harald Grosse and Carmelo P. Martín for their useful comments. C. T. wishes to thank the members of the Theoretical Physics Department at the Rudjer Bošković Institute, Zagreb, for their kind hospitality; his work received financial support from MICINN through grant FIS2005-02309. The work of J. T. is supported by the Croatian Ministry of Science Education and Sports project No. 098-0982930-2900, and is in part supported by the EU (HEPTOOLS) project under contract MRTN-CT-2006-035505.

[1] J. Madore, S. Schraml, P. Schupp and J. Wess, "Gauge theory on noncommutative spaces," Eur. Phys. J. C 16 (2000) 161 [arXiv:hep-th/0001203]; B. Jurco, S. Schraml, P. Schupp and J. Wess, "Enveloping algebra valued gauge transformations for non-Abelian gauge groups on non-commutative spaces," Eur. Phys. J. C 17 (2000) 521 [arXiv:hep-th/0006246]; B. Jurco, L. Moller, S. Schraml, P. Schupp and J. Wess, "Construction of non-Abelian gauge theories on noncommutative spaces," Eur. Phys. J. C 21 (2001) 383 [arXiv:hep-th/0104153].

[2] N. Seiberg and E. Witten, "String theory and noncommutative geometry," JHEP 9909, 032 (1999) [arXiv:hep-th/9908142].

[3] X. Calmet, B. Jurco, P. Schupp, J. Wess and M. Wohlgenannt, "The standard model on non-commutative space-time," Eur. Phys. J. C 23 (2002) 363 [arXiv:hep-ph/0111115].

[4] W. Behr, N. G. Deshpande, G. Duplancic, P. Schupp, J. Trampetic and J. Wess, "The $Z \rightarrow \gamma \gamma / g g$ decays in the noncommutative standard model," Eur. Phys. J. C 29 (2003) 441 [arXiv:hep-ph/0202121]; G. Duplancic, P. Schupp and J. Trampetic, "Comment on triple gauge boson interactions in the non-commutative electroweak sector," Eur. Phys. J. C 32 (2003) 141 [arXiv:hep-ph/0309138]. 
[5] B. Melic, K. Passek-Kumericki, J. Trampetic, P. Schupp and M. Wohlgenannt, "The standard model on non-commutative space-time: Electroweak currents and Higgs sector," Eur. Phys. J. C 42 (2005) 483 [arXiv:hep-ph/0502249]; "The standard model on non-commutative space-time: Strong interactions included," Eur. Phys. J. C 42 (2005) 499 [arXiv:hep$\mathrm{ph} / 0503064]$.

[6] P. Aschieri, B. Jurco, P. Schupp and J. Wess, "Non-commutative GUTs, standard model and C, P, T," Nucl. Phys. B 651, 45 (2003) [arXiv:hep-th/0205214].

[7] M. Buric, V. Radovanovic and J. Trampetic, "The one-loop renormalization of the gauge sector in the noncommutative standard model," JHEP 0703 (2007) 030 [arXiv:hep-th/0609073].

[8] D. Latas, V. Radovanovic and J. Trampetic, "Non-commutative SU(N) gauge theories and asymptotic freedom," Phys. Rev. D 76 (2007) 085006 [arXiv:hep-th/0703018].

[9] C. P. Martin, "The gauge anomaly and the Seiberg-Witten map," Nucl. Phys. B 652 (2003) 72 [arXiv:hep-th/0211164].

[10] F. Brandt, C. P. Martin and F. R. Ruiz, "Anomaly freedom in Seiberg-Witten noncommutative gauge theories," JHEP 0307, 068 (2003) [arXiv:hep-th/0307292].

[11] M. Buric, D. Latas, V. Radovanovic and J. Trampetic, "The absence of the $4 \psi$ divergence in noncommutative chiral models," Phys. Rev. D 77 (2008) 045031 [arXiv:0711.0887 [hep-th]].

[12] B. Melic, K. Passek-Kumericki and J. Trampetic, "Quarkonia decays into two photons induced by the space-time noncommutativity," Phys. Rev. D 72, 054004 (2005) [arXiv:hep-ph/0503133].

[13] B. Melic, K. Passek-Kumericki and J. Trampetic, " $K \rightarrow \pi \gamma$ decay and space-time noncommutativity," Phys. Rev. D 72 (2005) 057502 [arXiv:hep-ph/0507231].

[14] A. Alboteanu, T. Ohl and R. Ruckl, "Probing the noncommutative standard model at hadron colliders," Phys. Rev. D 74 (2006) 096004 [arXiv:hep-ph/0608155]; "The Noncommutative Standard Model at $O\left(\theta^{2}\right)$," Phys. Rev. D 76 (2007) 105018 [arXiv:0707.3595 [hep-ph]]; J. Trampetic, "Renormalizability and Phenomenology of theta-expanded Noncommutative Gauge Field Theory," Fortsch. Phys. 56, 521 (2008) [arXiv:0802.2030 [hep-ph]]; J. A. Conley and J. L. Hewett, "Effects of the Noncommutative Standard Model on WW scattering," arXiv:0811.4218 [hep-ph].

[15] M. Buric, D. Latas, V. Radovanovic and J. Trampetic, "Nonzero $Z \rightarrow \gamma \gamma$ decays in the renormalizable gauge sector of the noncommutative standard model," Phys. Rev. D 75 (2007) 097701 [arXiv:hep-ph/0611299].

[16] P. Schupp, J. Trampetic, J. Wess and G. Raffelt, "The photon neutrino interaction in non-commutative gauge field theory and astrophysical bounds," Eur. Phys. J. C 36, 405 (2004) [arXiv:hep-ph/0212292].

[17] C. P. Martin and C. Tamarit, "Renormalisability of the matter determinants in noncommutative gauge theory in the enveloping-algebra formalism," Phys. Lett. B 658, 170 (2008) [arXiv:0706.4052 [hep-th]].

[18] H. Grosse and G. Lechner, "Noncommutative Deformations of Wightman Quantum Field Theories," JHEP 0809 (2008) 131 [arXiv:0808.3459 [math-ph]].

[19] J. M. Grimstrup and R. Wulkenhaar, "Quantisation of theta-expanded non-commutative QED," Eur. Phys. J. C 26, 139 (2002) [arXiv:hep-th/0205153].

[20] C. Amsler et al. [Particle Data Group], "Review of particle physics," Phys. Lett. B 667 (2008) 1.

[21] N. Brambilla et al. [Quarkonium Working Group], "Heavy quarkonium physics," FERMILAB-FN-0779, CERN-2005005, Dec 2004. 521pp. Published as CERN Yellow Report, CERN-2005-005, Geneva: CERN, 2005. -487 p. arXiv:hep$\mathrm{ph} / 0412158$.

[22] L. Alvarez-Gaume and M. A. Vazquez-Mozo, "General properties of noncommutative field theories," Nucl. Phys. B 668 (2003) 293 [arXiv:hep-th/0305093]; S. A. Abel, J. Jaeckel, V. V. Khoze and A. Ringwald, "Vacuum birefringence as a probe of Planck scale noncommutativity," JHEP 0609 (2006) 074 [arXiv:hep-ph/0607188].

[23] A. Bichl, J. Grimstrup, H. Grosse, L. Popp, M. Schweda and R. Wulkenhaar, "Renormalization of the noncommutative photon self-energy to all orders via Seiberg-Witten map," JHEP 0106 (2001) 013 [arXiv:hep-th/0104097].

[24] S. Klous [LHCb Collaboration], "The LHCb vertex detector," Nucl. Instrum. Meth. A 549 (2005) 55. 